\title{
Hyperscaling-violating Lifshitz hydrodynamics from black-holes: part II
}

\author{
Elias Kiritsis $^{a, b, c, 1}$ and Yoshinori Matsuo ${ }^{d}$ \\ ${ }^{a}$ Crete Center for Theoretical Physics, Institute of Theoretical and Computational Physics, \\ Department of Physics, University of Crete, 71003 Heraklion, Greece \\ ${ }^{b}$ Crete Center for Quantum Complexity and Nanotechnology, \\ Department of Physics, University of Crete, 71003 Heraklion, Greece \\ ${ }^{c}$ APC Univ Paris Diderot, Sorbonne Paris Cité, \\ UMR 7164 CNRS, F-75205 Paris, France \\ ${ }^{d}$ Department of Physics, National Taiwan University, \\ Taipei 10617, Taiwan, R.O.C. \\ E-mail: matsuo@phys.ntu.edu.tw
}

ABSTRACT: The derivation of Lifshitz-invariant hydrodynamics from holography, presented in [1] is generalized to arbitrary hyperscaling violating Lifshitz scaling theories with an unbroken $\mathrm{U}(1)$ symmetry. The hydrodynamics emerging is non-relativistic with scalar "forcing". By a redefinition of the pressure it becomes standard non-relativistic hydrodynamics in the presence of specific chemical potential for the mass current. The hydrodynamics is compatible with the scaling theory of Lifshitz invariance with hyperscaling violation. The bulk viscosity vanishes while the shear viscosity to entropy ratio is the same as in the relativistic case. We also consider the dimensional reduction ansatz for the hydrodynamics and clarify the difference with previous results suggesting a non-vanishing bulk viscosity.

KEYwords: AdS-CFT Correspondence, Black Holes, Holography and condensed matter physics (AdS/CMT)

ARXiv EPrint: 1611.04773

\footnotetext{
${ }^{1}$ http://hep.physics.uoc.gr/ kiritsis/.
} 


\section{Contents}

1 Introduction, results and outlook 1

2 Hyperscaling-violating solutions and black holes 5

$\begin{array}{lll}3 & \text { Solving the hydrodynamics ansatz } & 7\end{array}$

4 Calculation of the stress tensor $\quad 11$

5 Hyperscaling-violating Lifshitz hydrodynamics $\quad 14$

$\begin{array}{ll}5.1 \text { Thermodynamics } & 14\end{array}$

5.2 Relation to hydrodynamics in Newton-Cartan theory 15

$\begin{array}{ll}5.3 \text { The entropy current } & 20\end{array}$

6 The relation to dimensional reduction $\quad 20$

6.1 Higher-dimensional thermodynamics and hydrodynamics 24

$\begin{array}{ll}\text { 6.2 Thermodynamics after the dimensional reduction } & 27\end{array}$

$\begin{array}{ll}6.3 \text { Hydrodynamics after the dimensional reduction } & 28\end{array}$

$7 \quad$ Lifshitz hydrodynamics on conformally flat background $\quad 31$

7.1 Dimensional reduction for conformally flat background 33

$\begin{array}{ll}\text { A Notations } & 34\end{array}$

B The Newton-Cartan formalism $\quad 36$

$\begin{array}{ll}\text { C Lifshitz fluid in scalar background } & 41\end{array}$

$\begin{array}{ll}\text { D Scaling dimensions in fluids } & 43\end{array}$

\section{Introduction, results and outlook}

Holography $[2-5]$ is a correspondence between quantum field theory and gravity and provides a powerful tool to analyze strong coupling theories by using the dual description of gravity. At finite temperature and in the long wavelength regime, holography provides a correspondence between fluids and black holes. This fluid/gravity correspondence was first studied in the AdS/CFT correspondence by [6-9] and a more intuitive description was studied in [10].

Holography has also been applied to geometries with Lifshitz or Schrödinger symmetry mainly for application to condensed matter systems [12-19]. ${ }^{1}$ Many condensed matter

\footnotetext{
${ }^{1}$ For a recent review see [20].
} 
systems have non-relativistic scale invariance [21-26], and some of them have Lifshitz or Schrödinger symmetry [27-29]. Moreover, the hydrodynamics of charge and energy in such systems may be interesting as has been argued recently for the case of cold fermions at unitarity, [35], other strongly correlated systems, [36] and graphene, [37-39]. Recent experiments in various materials that are strongly coupled as well as graphene have indicated that the hydrodynamics of electrons is observable and it exhibits non-trivial shear viscosity, [40-45].

The fluid/gravity correspondences in Schrödinger spacetimes has been studied in [3032], where the dual description for non-relativistic fluid mechanics was given. A proposal for hydrodynamics in Lifshitz invariant theories was considered in [33, 34] from the effective field theory point of view.

In [17-19], all quantum critical holographic scaling geometries with a U(1) symmetry respecting translation invariance and spatial rotation invariance were classified in terms of three scaling exponents. Two of them $(z, \theta)$ appear in the metric while another exponent, which is referred to as $\zeta$ in [17-19], appears in the profile of the U(1) gauge field. ${ }^{2}$ The exponent $z$ is the Lifshitz (dynamical) scaling exponent, and $\theta$ is the hyperscaling violation exponent, [18, 49]. Even though such theories have been studied intensively, many of the aspects are still unclear. In particular, hydrodynamics with Lifshitz scaling symmetry is not fully understood.

In non-relativist quantum field theories that geometry that captures the dynamics is the Newton Cartan geometry, [50]. For Lifshitz space-times, the dual field theory is non-relativistic and the source terms at the boundary are related to the torsional NewtonCartan theory [51-56]. In particular, the role of torsional Newton-Cartan geometry in the boundary structure of bulk non-relativistic solutions as well as on the boundary symmetries was investigated in [54-56]. Alternatively the boundary structure can be analyzed using the Hamilton-Jacobi method, [57-59].

In [1], the correspondence between fluids in the torsional Newton-Cartan theory and black holes in Lifshitz space-times has been analyzed. Lifshitz space-times with unbroken U(1) gauge symmetry were considered, that were solutions of the Einstein-Maxwell-dilaton (EMD) theories with constant potential. Although the geometry has Lifshitz scaling symmetry with dynamical exponent $z$, there was a non-trivial gauge field in the bulk that introduced non-trivial scaling exponents in the charged sector. The black-hole solution of that theory was considered, boosted using Galilean boosts and then all parameters of the solution including the velocities, were made $\vec{x}$-dependent. Using the technique introduced in [10], the bulk equations of motion were solved order by order in boundary derivatives and the (fluid) stress-energy tensor was computed and renormalized. The results founds were as follows:

- The stress-energy tensor was expressed in terms of the fluid variables: velocity field $v^{i}$, energy density $\mathcal{E}$ and pressure $P$, but it also contained the (particle number) density

\footnotetext{
${ }^{2}$ This charge exponent controls the anomalous scaling of the charge density, even if it is conserved. It has also been introduced independently in [46] and was studied in more detail in [47] and [48]. The reason for the existence of anomalous charge exponent despite conservation is the RG running of the bulk coupling for charged degrees of freedom.
} 
$n$ and external source $\mathcal{A}_{i}$ associated to the $\mathrm{U}(1)$ symmetry current. It satisfied the scaling condition for Lifshitz invariant theories

$$
z \mathcal{E}=(d-1) P
$$

- It was found that the stress tensor satisfied the conservation equations of the NewtonCartan theory.

- The role of the (unbroken) U(1) symmetry in this class of theories is important. It was found that it behaves very closely to the $\mathrm{U}(1)$ mass conservation symmetry in non-relativistic hydrodynamics.

- The fluid equations found were non-relativistic in contrast to the relativistic fluid analysis in [33, 34]. Even though the continuity equation and energy conservation equation agree with those in the ordinary non-relativistic fluids, the Navier-Stokes equation was different from that in the ordinary non-relativistic fluids.

- By redefining variables and allowing a (Milne-invariant) Newton potential in the sources, [54]-[56] the fluid equations can be mapped to the standard non-relativistic fluid equations coupled to the torsional Newton-Cartan geometry in the presence of a Newton potential.

- It was found that the form of the fluid equations is independent of the Lifshitz exponent $z$ as well as of the (non-trivial) conduction exponent. It is only the constitutive relations (equation of state) that depend on these scaling exponents.

- The entropy satisfies the local thermodynamic relation with the energy density and pressure. The divergence of the entropy current is non-negative, compatible with the second law.

The hydrodynamics analysis of [1] was generalized beyond the hydrodynamic limit in [60] using numerical techniques.

In this paper, we generalize the studies in [1] to those with hyperscaling-violation in the associated holographic geometries. In [1], we have considered only the $\theta=0$ cases in which hyperscaling-violation comes only from the gauge field. Here, we introduce a nontrivial coupling between the dilaton and cosmological constant in order to consider $\theta \neq 0$. Fluids now, have hyperscaling-violation. We find the following results:

- As in [1], the fluid equations and the stress-energy tensor reproduce those in the Newton-Cartan theory if the gauge field is identified to that in the Newton-Cartan theory.

- The fluid equations are similar to those in [1] but for additional external forcing terms which come from the coupling to the dilaton and its external source term. Note however that in the Lifshitz case with $\theta=0$, although the dilaton was nontrivial, no such terms appeared in the hydrodynamics. Their appearance is therefore tied to hyperscaling violation in the bulk geometry. 
- With a judicious choice of the pressure, these forcing terms can be redefined away. The hydrodynamic equations are now equivalent to the non-relativistic hydrodynamics equations with a conserved mass current, but with an additional chemical potential for the mass current, that is given in (5.8). This chemical potential is not related to external sources like a Newtonian potential but is directly related to the hyperscaling violation.

- The bulk viscosity is zero even in the presence of hyperscaling-violation.

- The hydrodynamic equations respect the full scaling symmetry of Lifshitz invariance with hyperscaling violation as detailed in appendix D

The Lifshitz space-time with hyperscaling-violation can be also obtained by dimensional reduction from higher-dimensional Lifshitz space-times without hyperscaling violation, [18]. In this case, the model has two dilatons. We derive also the associated hydrodynamics from the black holes. We find the following:

- The bulk viscosity is non-zero if we consider the naive dimensional reduction, i.e., with trivial background in the extra dimensions and fixed volume.

- When the internal metric on the extra dimensions, or equivalently, the volume of the extra dimensions satisfies a specific relation to the $\mathrm{U}(1)$ charge, the bulk viscosity becomes zero. This reduction gives the lower dimensional hydrodynamics we derived earlier.

- We also present the hydrodynamic ansatz which describes a Lifshitz-invariant fluid with hyperscaling violation on a non-trivial (conformally-flat) boundary metric. The hydrodynamic equations in such a metric turn out to be simpler than on flat space.

The hydrodynamic equations we derive are general and are given by (5.52)-(5.54) and supplemented by (5.56), with independent variables the temperature $T$, the (mass) charge density $n$ and velocities $v^{i}$. All other variables like the energy $\mathcal{E}$, the pressure $P$ and the chemical potential as well as the transport coefficients are determined in terms of $T, n$ by constitutive relations that are dynamics/theory specific. ${ }^{3}$ Finally there is the Lifshitz invariance Ward identity (5.55).

The $z, \theta$ dependence does appear explicitly in the hydrodynamic equations. To leading order, it appears only through the constituent relations that express the energy, pressure and (mass) chemical potential as functions of temperature and mass density. To next order the transport coefficients may also depend on $z$ and $\theta$. For this reason we expect that although the hydrodynamic equations we derived strictly apply to the duals of EMD theories we considered, their validity is universal.

\footnotetext{
${ }^{3}$ Here, $n$ plays the role of the density of the non-relativistic fluid. We use this (and not the associated chemical potential) as a fluid variable because of the important role it plays in the realization of the nonrelativistic momenta. It is the same reason that the mass density is used usually in non-relativistic fluid mechanics.
} 
This analysis completes the derivation of fluid dynamics for non-relativistic scaleinvariant and U(1)-invariant theories with Lifshitz scaling and a violation of hyperscaling. Remains open the case of such theories without a U(1) symmetry (ie. a broken U(1) symmetry). The case of a perfect fluid was studied recently in [61]. It is highly probable that the hydrodynamics in this case will turn out to be non-relativistic hydrodynamics in the absence of a conserved "mass" current.

A further interesting question concerns the appropriate hydrodynamics for QFTs that are described by RG flows that interpolate between relativistic and non-relativistic theories. To motivate the answer to this question, we consider first non-Lorentz invariant (but rotationally invariant) flows between Lorentz invariant fixed points, ${ }^{4}[63,64]$, but where the velocity of light in the IR is different for that in the UV. In such a case, the hydrodynamics of this theory, is quasi-relativistic, but with a speed of light that is temperature dependent. This context resembles more the proposal of [33, 34].

The example above suggests that in a (Lorentz-violating) RG flow from a CFT (with a un unbroken $\mathrm{U}(1)$ symmetry that is used to drive the breaking of Lorentz invariance) to an IR non-relativistic scaling (rotational invariant) geometry at an arbitrary temperature, the hydrodynamics will be again of the relativistic form (but with general equation of state) and with a speed of light $c(T)$ that is again temperature dependent. In the IR, $c(T \rightarrow 0)=\infty$ and the hydrodynamics reduces to the one found here with the U(1) symmetry becoming the mass-related symmetry. This is the standard non-relativistic limit of the relativistic hydrodynamics while all thermodynamic functions and transport coefficients are smooth functions of $T$ (If no phase transition exists at finite $T$ ). Otherwise they follow the standard behavior at phase transitions.

More general breaking of Lorentz invariance during RG flow must involve higher form fields of tensors in the bulk, and the details of the RG flow become complicated. It is plausible that a RG-covariant definition of velocities and other state functions is necessary in order to define hydrodynamics globally in the full energy landscape. ${ }^{5}$

This paper is organized as follows. In section 2, we introduce the model and its asymptotically Lifshitz solution with hyperscaling-violation. In section 3 , we introduce the hydrodynamic ansatz. We consider the derivative expansion and solve the equations of motion at the first order. We calculate the stress-energy tensor in section 4, and discuss relation to the non-relativistic fluids in section 5 . In section 6 , we consider the relation to the dimensional reduction from higher dimensional Lifshitz space-times (without hyperscalingviolation). In section 7 , we show that a simpler hydrodynamic ansatz gives fluid in nontrivial but conformally flat background. In appendix B, we will review on the NewtonCartan theory and fluids. In appendix D, we will discuss the scaling dimension of the fluid variables and coefficients.

\section{Hyperscaling-violating solutions and black holes}

We consider a holographic model with Lifshitz scaling symmetry and hyperscalingviolation. The gravity dual is $(d+1)$-dimensional Einstein gravity with a Maxwell field $A_{\mu}$

\footnotetext{
${ }^{4}$ The fact that the speed off light can vary on branes was pointed out first in [62].

${ }^{5} \mathrm{~A}$ proposal was put forward in [65-67] but we think the appropriate setup is a bit different.
} 
and a dilaton $\phi$. The action is given by

$$
S=\frac{1}{16 \pi G} \int d^{d+1} x \sqrt{-g}\left(R-2 \Lambda e^{-\nu \phi}-\frac{1}{2}(\partial \phi)^{2}-\frac{1}{4} e^{\lambda \phi} F^{2}\right),
$$

where $F=d A$ is the field strength of the gauge field, $\Lambda$ is a negative cosmological constant with coupling to dilaton, and $\lambda$ and $\nu$ are dimensionless coupling constants. The equations of motion are given by

$$
\begin{aligned}
R_{\mu \nu} & =\frac{2 \Lambda}{d-1} e^{-\nu \phi} g_{\mu \nu}+\frac{1}{2}\left(\partial_{\mu} \phi\right)\left(\partial_{\nu} \phi\right)+\frac{1}{4} e^{\lambda \phi}\left(2 F_{\mu \rho} F_{\nu}{ }^{\rho}-\frac{1}{d-1} F^{2} g_{\mu \nu}\right) \\
0 & =\nabla_{\mu}\left(e^{\lambda \phi} F^{\mu \nu}\right), \\
\square \phi & =\frac{1}{4} \lambda e^{\lambda \phi} F^{2}-2 \nu \Lambda e^{-\nu \phi} .
\end{aligned}
$$

This model has the Lifshitz geometry with hyperscaling-violation as a solution;

$$
\begin{aligned}
d s^{2} & =e^{2 \chi} d \tilde{s}^{2}, \\
d \tilde{s}^{2} & =-r^{2 z} d t^{2}+\frac{d r^{2}}{r^{2}}+\sum_{i} r^{2}\left(d x^{i}\right)^{2}, \\
e^{\chi} & =e^{\chi 0} r^{-\theta /(d-1)}
\end{aligned}
$$

The solution for the gauge field and dilaton are given by

$$
A_{t}=a \sqrt{\mu} r^{z+d-1-\theta}, \quad \quad e^{\lambda \phi}=e^{\lambda \phi_{0}} r^{-2 d_{1}} .
$$

where

$$
d_{1}=(d-1)-\frac{d-2}{d-1} \theta
$$

Here, the cosmological constant is related to the radius of the geometry, which we fixed to be 1 , and then, $\Lambda$ is given by

$$
\Lambda=-\frac{(z-d-1-\theta)(z+d-2-\theta)}{2} .
$$

The exponents $z, \theta$ and constants $\phi_{0}, \chi_{0}, \mu$ are determined by the coupling constants in the action $\lambda, \nu$, and the free parameter $a$ of the solution by the following relations;

$$
\begin{aligned}
\lambda^{2} & =2 \frac{(d-1) d_{1}^{2}}{[(z-1)(d-1)-\theta][(d-1)-\theta]}, \\
\nu & =\frac{\theta \lambda}{(d-1) d_{1}}, \\
\phi_{0} & =-\frac{2}{\lambda}\left(1+\frac{\theta}{(d-1)(d-1-\theta)}\right) \log a, \\
\chi_{0} & =-\frac{\theta}{(d-1)(d-1-\theta)} \log a, \\
\mu & =\frac{2(z-1)}{z+d-1-\theta} .
\end{aligned}
$$


To simplify the calculation, we rescale the gauge field such that the solution becomes

$$
A_{t}=a r^{z+d-1-\theta},
$$

and then the action becomes

$$
S=\frac{1}{16 \pi G} \int d^{d+1} x \sqrt{-g}\left(R-2 \Lambda e^{-\nu \phi}-\frac{1}{2}(\partial \phi)^{2}-\frac{\mu}{4} e^{\lambda \phi} F^{2}\right) .
$$

The following black hole geometry is also a solution of this model;

$$
\begin{aligned}
d s^{2} & =e^{2 \chi} d \tilde{s}^{2} \\
d \tilde{s}^{2} & =-r^{2 z} f(r) d t^{2}+\frac{d r^{2}}{f(r) r^{2}}+\sum_{i} r^{2}\left(d x^{i}\right)^{2}, \\
e^{\chi} & =e^{\chi_{0}} r^{-\theta /(d-1)}
\end{aligned}
$$

where

$$
f=1-\frac{r_{0}^{z+d-1-\theta}}{r^{z+d-1-\theta}}
$$

The radius of the horizon is given by $r_{0}$ and the Hawking temperature of the black hole is

$$
T=\frac{z+d-1-\theta}{4 \pi} r_{0}^{z} .
$$

The gauge field and dilaton take almost the same form as in the the zero temperature solution

$$
A_{t}=a\left(r^{z+d-1-\theta}-r_{0}^{z+d-1-\theta}\right), \quad \quad e^{\lambda \phi}=e^{\lambda \phi_{0}} r^{-2 d_{1}} .
$$

but the constant mode of $A_{t}$ is chosen such that $A_{t}$ vanishes at the horizon for the regularity there.

\section{Solving the hydrodynamics ansatz}

In this paper, we focus on the case of $d=4$. In some parts we will give results in arbitrary dimensions. Extensions to other dimensions (namely $d=3$ and $d>4$ ) are expected to be straightforward.

We introduce the hydrodynamic ansatz, which describes the fluid mechanics in the field theory side. For the regularity at the future horizon, we take the Eddington-Finkelstein coordinates;

$$
d t_{+}=d t+\frac{d r}{r^{z+1} f} .
$$

Hereafter, we always take the Eddington-Finkelstein coordinates and $t$ will stands for the null coordinate $t_{+}$. In this coordinates, the black hole solution for the metric and gauge field are expressed as

$$
\begin{aligned}
d s^{2} & =r^{2 \chi} d \tilde{s}^{2} \\
d \tilde{s}^{2} & =-r^{2 z} f d t^{2}+2 r^{z-1} d t d r+r^{2}\left(d x^{i}\right)^{2}, \\
A & =a\left(r^{z+3-\theta}-r_{0}^{z+3-\theta}\right) d t-a r^{2-\theta} d r
\end{aligned}
$$


where we have taken the gauge such that $A_{r}$ vanishes in the Fefferman-Graham coordinates. Then, we introduce the Galilean boost

$$
t \rightarrow t, \quad x^{i} \rightarrow x^{i}-v^{i} t,
$$

where $v^{i}$ is a constant parameter of the Galilean boost. Note that this is a general coordinate transformation and for constant $v^{i}$ it provides a new class of solutions parametrized by $\vec{v}$. Moreover, we assume in this paper $^{6}$ that $z \neq 1$ and therefore such a coordinate transformation keeps the boundary sources ${ }^{7}$ invariant, as it should. It therefore provides a different state (more precisely ensemble) of the theory.

The black hole geometry becomes

$$
\begin{aligned}
d s^{2} & =e^{2 \chi} d \tilde{s}^{2}, \\
d \tilde{s}^{2} & =-\left(r^{2 z} f-v^{2} r^{2}\right) d t^{2}+2 r^{z-1} d t d r-2 r^{2} v^{i} d t d x^{i}+r^{2}\left(d x^{i}\right)^{2}, \\
e^{\chi} & =e^{\chi 0} r^{-\theta / 3}
\end{aligned}
$$

and the solution for the gauge field and dilaton remains invariant;

$$
A=a\left(r^{z+3-\theta}-r_{0}^{z+3-\theta}\right) d t-a r^{2-\theta} d r, \quad e^{\lambda \phi}=e^{\lambda \phi_{0}} r^{-2 d_{1}} .
$$

In order to describe the dynamics of the fluid, we replace the free parameters $r_{0}, v^{i}$ and $a$ by slowly varying functions of the boundary coordinates $(\vec{x}, t)$. However, this procedure generates a non-trivial background metric at the boundary since $a$ appears as an overall factor of the metric. In order to obtain a flat space background at the boundary, we absorb this overall factor by introducing the additional coordinate transformation

$$
x^{\mu} \rightarrow e^{-\chi_{0}} x^{\mu}
$$

with $\chi_{0}$ given in (2.14), before replacing the parameters by functions. Then, the metric becomes

$$
d s^{2}=r^{-2 \theta / 3}\left[-\left(r^{2 z} f-v^{2} r^{2}\right) d t^{2}+2 e^{\chi_{0}} r d t d r-2 r^{2} v^{i} d t d x^{i}+r^{2}\left(d x^{i}\right)^{2},\right]
$$

and the gauge field is also rescaled as

$$
A=a^{1+\frac{\theta}{3(3-\theta)}}\left(r^{z+3-\theta}-r_{0}^{z+3-\theta}\right) d t-a r^{2-\theta} d r .
$$

Since we have rescaled the time coordinate, the Hawking temperature is also rescaled as

$$
T=\frac{z+d-1-\theta}{4 \pi} e^{-\chi_{0}} r_{0}^{z}=\frac{z+d-1-\theta}{4 \pi} a^{\frac{\theta}{(d-1)(d-1-\theta)}} r_{0}^{z} .
$$

Now, we replace the parameters $r_{0}, v^{i}$ and $a$ by slowly varying function of the boundary coordinates $x^{\mu}$. Moreover, we promote the constant part of $A_{i}$, which is usually gauged

\footnotetext{
${ }^{6}$ The hydrodynamics of $z=1$ with hyperscaling violation was considered in [72] obtaining such solutions by dimensional reduction from higher dimensional AdS geometries.

${ }^{7}$ They are defined as the most divergent parts of the metric and gauge field near the boundary.
} 
away to $x^{\mu}$-dependent functions as was also done in [1]. Then, the metric, gauge field and dilaton become

$$
\begin{aligned}
d s^{2} & =r^{-2 \theta / 3}\left[-r^{2 z} f d t^{2}+2 e^{\chi_{0}(x)} r d t d r+r^{2}\left(d x^{i}-v^{i}(x) d t\right)^{2}\right] \\
f & =1-\frac{r_{0}^{z+3-\theta}(x)}{r^{z+3-\theta}}, \\
A & =a^{1+\frac{\theta}{3(3-\theta)}}(x)\left(r^{z+3-\theta}-r_{0}^{z+3-\theta}(x)\right) d t-a(x) r^{2-\theta} d r+\mathcal{A}_{\mu}(x) d x^{\mu}, \\
e^{\lambda \phi} & =e^{\lambda \phi_{0}(x)} r^{-2 d_{1}}
\end{aligned}
$$

where $x^{\mu}$-dependence of $\phi_{0}(x)$ and $\chi_{0}(x)$ comes from that of $a(x)$. We have also introduced $\mathcal{A}_{t}(x)$ and $\mathcal{A}_{i}(x)$, that originate in the constant parts of $A_{t}$ and $A_{i}$, respectively, and which have been now replaced by functions of the boundary coordinates. The relations (2.13)(2.15) are however preserved. The above are no longer a solution of the equations of motion, and we must introduce additional correction terms.

We consider the derivative expansion in $t, \vec{x}$ and calculate the first order solution for the hydrodynamic ansatz. We first expand (3.14)-(3.17) at a point, which we can take $x^{\mu}=0$ without loss of generality. Then, we assume that the derivatives are small since $x^{\mu}$ dependence appears only through the slowly varying functions $r_{0}(x), v^{i}(x), a(x)$, etc., and expand with respect to the derivatives $\partial_{\mu}$;

$$
\begin{aligned}
g_{\mu \nu} & =g_{\mu \nu}^{(0)}+g_{\mu \nu}^{(1)}+\cdots, \\
A_{\mu} & =A_{\mu}^{(0)}+A_{\mu}^{(1)}+\cdots, \\
\phi & =\phi^{(0)}+\phi^{(1)}+\cdots,
\end{aligned}
$$

where $g_{\mu \nu}^{(n)}$, etc. stands for $n$-th order terms in the derivative expansion. At the leading order of the derivative expansion, the equations of motion contain only the leading order terms $g_{\mu \nu}^{(0)}, A_{\mu}^{(0)}$ and $\phi^{(0)}$ which are equivalent to the solutions (3.6)-(3.9) before replacing the parameters by slowly varying functions, and hence, are satisfied. At the next-to-leading order, only the linear order terms of the derivatives $\partial_{\mu}$ appear, and the equations of motion are not satisfied due to these linear order terms. Now, we introduce the correction terms to $(3.14)-(3.17)$ as

$$
\begin{aligned}
g_{\mu \nu} & =g_{\mu \nu}^{(0)}+g_{\mu \nu}^{(1)}+h_{\mu \nu}^{(1)}+\mathcal{O}\left(\partial^{2}\right), \\
A_{\mu} & =A_{\mu}^{(0)}+A_{\mu}^{(1)}+a_{\mu}^{(1)}+\mathcal{O}\left(\partial^{2}\right), \\
\phi & =\phi^{(0)}+\phi^{(1)}+\varphi^{(1)}+\mathcal{O}\left(\partial^{2}\right),
\end{aligned}
$$

where $h_{\mu \nu}^{(n)}, a_{\mu}^{(n)}$ and $\varphi^{(n)}$ are the correction terms at the $n$-th order of the derivative expansion, which start from $n=1$ since the equations of motion satisfied without the correction terms at the leading order. At the next-to-leading order, or equivalently, at the linear order of the derivative expansion, the equations of motion give the inhomogeneous linear differential equations for the correction terms, $h_{\mu \nu}^{(1)}, a_{\mu}^{(1)}$ and $\varphi^{(1)}$. The inhomogeneity comes from the first order terms of (3.14)-(3.17), namely, $g_{\mu \nu}^{(1)}, A_{\mu}^{(1)}$ and $\phi^{(1)}$. 
By solving the differential equations for the correction terms, we obtain the following first order solution of the derivative expansion;

$$
\begin{aligned}
d s^{2}=r^{-2 \theta / 3}[- & r^{2 z} f d t^{2}+2 a^{-\frac{\theta}{3(3-\theta)}} r^{z-1} d t d r+r^{2}\left(d x^{i}-v^{i} d t\right)^{2} \\
& +\frac{2}{3-\theta} a^{-\frac{\theta}{3(3-\theta)}} r^{z} \partial_{i} v^{i} d t^{2}-r^{2} F_{1}(r) \sigma_{i j}\left(d x^{i}-v^{i} d t\right)\left(d x^{j}-v^{j} d t\right) \\
& \left.+2\left(F_{3}(r) \partial_{i} r_{0}+F_{5}(r) \partial_{i} a\right) d t\left(d x^{i}-v^{i} d t\right)\right]
\end{aligned}
$$

where the first line is the original solution and the the rest are the corrections terms. $\sigma_{i j}$ is the shear tensor

$$
\sigma_{i j}=\left(\partial_{i} v^{j}+\partial_{j} v^{i}\right)-\frac{2}{3} \partial_{k} v^{k} \delta_{i j},
$$

and the functions $F(r)$ are given by

$$
\begin{aligned}
& F_{1}(r)=a^{-\frac{\theta}{3(3-\theta)}} \int_{\infty}^{r} d r^{\prime} \frac{r^{\prime 3-\theta}-r_{0}^{3-\theta}}{r^{\prime}\left(r^{\prime z+3-\theta}-r_{0}^{z+3-\theta}\right)}, \\
& F_{2}(r)=\left(2(z-1) r^{z+3-\theta}-(z-5+\theta) r_{0}^{z+3-\theta}\right) \int_{\infty}^{r} d r^{\prime} \widehat{F}_{1}\left(r^{\prime}\right) \\
& F_{3}(r)=-2(z-1) a^{-1-\frac{\theta}{3(3-\theta)}} \int_{\infty}^{r} \frac{d r^{\prime}}{r^{\prime 6-z+\theta}} F_{2}\left(r^{\prime}\right) \\
& F_{4}(r)=\left(2(z-1) r^{z+3-\theta}-(z-5+\theta) r_{0}^{z+3-\theta}\right) \int_{\infty}^{r} d r^{\prime} \widehat{F}_{2}\left(r^{\prime}\right), \\
& F_{5}(r)=\int_{\infty}^{r} \frac{d r^{\prime}}{r^{\prime 6-z+\theta}}\left(-2(z-1) a^{-1-\frac{\theta}{3(3-\theta)}} F_{4}\left(r^{\prime}\right)+\frac{\theta}{3(3-\theta)} a^{-1-\frac{\theta}{3(3-\theta)}} r^{\prime 3-\theta}\right) \\
& \widehat{F}_{i}(r)=\frac{r^{7-2 \theta} \widetilde{F}_{i}}{\left(r^{z+3-\theta}-r_{0}^{z+3-\theta}\right)\left[2(z-1) r^{z+3-\theta}-(z-5+\theta) r_{0}^{z+3-\theta}\right]^{2}} \\
& \widetilde{F}_{1}(r)=\frac{z+3-\theta}{2(z-1)} a \frac{r_{0}^{z-\theta}}{r^{5-\theta}}\left(2(z-1)(5-\theta) r^{z+3-\theta} r_{0}^{2}-z(z+3-\theta) r^{5-\theta} r_{0}^{z}\right. \\
& \left.\quad+(z-5+\theta)(z-2) r_{0}^{z+3-\theta}\right) \\
& \widetilde{F}_{2}(r)=-\frac{\theta}{6(3-\theta)(z-1)} r^{-\theta-5} r_{0}^{-2 \theta}\left(-4(z-1)^{2} r_{0}^{2 \theta} r^{2 z+6}-r^{2 \theta} r_{0}^{2 z+6}(z-5+\theta)^{2}\right. \\
& \left.\quad+r^{\theta+5}(z+3-\theta)^{2} r_{0}^{\theta+2 z+1}+4(z-1)(z-5+\theta) r_{0}^{\theta+z+3} r^{\theta+z+3}\right) .
\end{aligned}
$$

The first order solution of the gauge field is

$$
\begin{aligned}
A= & a(x)\left[a^{\frac{\theta}{3(3-\theta)}}\left(r^{z+3-\theta}-r_{0}^{z+3-\theta}(x)\right)-\frac{1}{3-\theta} r^{3-\theta} \partial_{i} v^{i}(x)\right] d t \\
& -a(x) r^{2-\theta} d r+\mathcal{A}_{\mu}(x) d x^{\mu}+\left(F_{2}(r) \partial_{i} r_{0}+F_{4}(r) \partial_{i} a\right)\left(d x^{i}-v^{i} d t\right),
\end{aligned}
$$

and the dilaton has no correction term,

$$
e^{\lambda \phi}=e^{\lambda \phi_{0}(x)} r^{-2 d_{1}},
$$


For (3.24), (3.34) and (3.35) to be a solution of the equations of motion, the functions $r_{0}(x), v^{i}(x), a(x)$ and $\mathcal{A}_{\mu}(x)$ must satisfy the following constrains;

$$
\begin{aligned}
& 0=\partial_{t} a+v^{i} \partial_{i} a-a \partial_{i} v^{i} \\
& 0=\partial_{t} r_{0}+v^{i} \partial_{i} r_{0}+\frac{1}{3-\theta} r_{0} \partial_{i} v^{i} \\
& 0=\mathcal{F}_{t i}+v^{j} \mathcal{F}_{j i}+\frac{z+3-\theta}{2(z-1)} a^{1+\frac{\theta}{3(3-\theta)}} r_{0}^{z+3-\theta}\left(z \frac{\partial_{i} r_{0}}{r_{0}}+\frac{\theta}{3(3-\theta)} \frac{\partial_{i} a}{a}\right)
\end{aligned}
$$

where $\mathcal{F}=d \mathcal{A}$.

\section{Calculation of the stress tensor}

We will calculate now the boundary stress-energy tensor. The asymptotically Lifshitz spacetimes have anisotropic behavior in temporal and spatial directions, and hence it is convenient to introduce the vielbeins in order to consider their general asymptotic behaviors.

The metric is expressed in terms of the vielbein $E^{A}$ as

$$
d s^{2}=-\left(E^{0}\right)^{2}+\delta_{a b} E^{a} E^{b}+\left(E^{r}\right)^{2} .
$$

For the asymptotic Lifshitz space-time with the Fefferman-Graham coordinates, the vielbein can be expressed as [68]

$$
E^{r}=e^{\chi} \frac{d r}{r}, \quad E_{\mu}^{0}=e^{\chi} r^{z} \tau_{\mu}\left(r, x^{\mu}\right), \quad E_{\mu}^{a}=e^{\chi} r \hat{e}_{\mu}^{a}\left(r, x^{\mu}\right),
$$

where the one forms $\tau\left(r, x^{\mu}\right)$ and $\hat{e}^{a}\left(r, x^{\mu}\right)$ have some finite and non-degenerate limit in $r \rightarrow$ $\infty$, which corresponds to the characteristic quantities of Newton Cartan geometry. Eq. (4.2) does not fix $r \rightarrow \infty$ limit of $\tau\left(r, x^{\mu}\right)$ and $\hat{e}^{a}\left(r, x^{\mu}\right)$, uniquely. For our solution (3.24), we take the vielbein $E^{A}$ such that $\tau\left(r, x^{\mu}\right)$ and $\hat{e}^{a}\left(r, x^{\mu}\right)$ behave in $r \rightarrow \infty$ as

$$
\tau_{\mu} d x^{\mu}=d t, \quad \hat{e}_{\mu}^{a} d x^{\mu}=d x^{a}-v^{a} d t .
$$

Then, the induced metric at surfaces $d r=0$ is expressed for large $r$ as

$$
\begin{aligned}
& \gamma_{\mu \nu}=r^{-2 \theta / 3}\left(-r^{2 z} \tau_{\mu} \tau_{\nu}+r^{2} \delta_{a b} \hat{e}_{\mu}^{a} \hat{e}_{\nu}^{b}\right), \\
& \gamma^{\mu \nu}=r^{2 \theta / 3}\left(-r^{-2 z} \hat{v}^{\mu} \hat{v}^{\nu}+r^{-2} \delta^{a b} e_{a}^{\mu} e_{b}^{\nu}\right),
\end{aligned}
$$

where $\hat{v}^{\mu}$ and $e_{a}^{\mu}$ are the inverse vielbeins, which take the following form ${ }^{8}$ in $r \rightarrow \infty$ for (3.24);

$$
\hat{v}^{\mu} \nabla_{\mu}=\nabla_{t}+v^{i} \nabla_{i}, \quad e_{a}^{\mu} \nabla_{\mu}=\nabla_{a} .
$$

Relations (4.4) and (4.5) do not completely fix the $r$-dependent vielbeins $\tau_{\mu} \hat{e}_{\mu}^{a}$ and $\hat{v}^{\mu}$. For example, the one form $\tau_{\mu}$ may also have subleading terms of order $\mathcal{O}\left(r^{2-2 z}\right)$, which

\footnotetext{
${ }^{8}$ Note that our notations is somewhat different from [54]-[56]. The detailed notation and variables used in summarized in appendix A.
} 
appear at the same order as the leading terms of $\hat{e}_{\mu}^{a}$. This would change $\hat{e}_{\mu}^{a}$ and $\hat{v}^{\mu}$ to leading order. This ambiguity is equivalent to a Milne boost at the boundary. We fix this ambiguity in the sequel in order to proceed. We will discuss the action of Milne boosts at the boundary data later.

To summarize, for our solution we take

$$
\tau=(1, \overrightarrow{0}) \quad, \quad \hat{v}=(1, \vec{v}) \quad, \quad \hat{e}_{\mu}^{a}=\left(\begin{array}{llll}
-v^{1} & 1 & 0 & 0 \\
-v^{2} & 0 & 1 & 0 \\
-v^{3} & 0 & 0 & 1
\end{array}\right) \quad, \quad e_{a}^{\mu}=\left(\begin{array}{lll}
0 & 0 & 0 \\
1 & 0 & 0 \\
0 & 1 & 0 \\
0 & 0 & 1
\end{array}\right)
$$

that describe the asymptotic Newton Cartan geometry in what we called in [1] as the holographic frame.

For the gauge field with boundary Lorentz indices we define

$$
\hat{A}_{0} \equiv \hat{v}^{\mu} A_{\mu}=A_{t}+\vec{v} \cdot \vec{A}, \quad \hat{A}_{a} \equiv e_{a}^{\mu} A_{\mu},
$$

The variation of the action with respect to these variables is given by

$$
\delta S_{r}=\int d^{4} x \sqrt{-\gamma}\left(-\hat{S}_{\mu}^{0} \delta \hat{v}^{\mu}+\hat{S}_{\mu}^{a} \delta e_{a}^{\mu}+\hat{J}^{0} \delta \hat{A}_{0}+\hat{J}^{a} \delta \hat{A}_{a}+\mathcal{O}_{\phi} \delta \phi\right),
$$

where $S_{r}$ is the renormalized action with appropriate (boundary) counter terms. Since the volume form behaves as

$$
\sqrt{-\gamma} \sim r^{z+3-4 \theta / 3}
$$

the terms at $\mathcal{O}\left(r^{-z-3+4 \theta / 3}\right)$ in the expectation values give the regular contributions. ${ }^{9}$

The definition above does not give the ordinary stress-energy tensor but the one with contributions from the gauge field and current. It is related to the ordinary Brown-York tensor as

$$
\hat{S}_{\nu}^{0} \hat{v}^{\mu}-\hat{S}_{\nu}^{a} e_{a}^{\mu}=T_{(\mathrm{BY})}{ }^{\mu}{ }_{\nu}+J^{\mu} A_{\nu}+T_{(\mathrm{ct})}{ }^{\mu}{ }_{\nu}
$$

where $T_{(\mathrm{BY})}^{\mu \nu}$ is the Brown-York tensor which is defined in terms of the extrinsic curvature $K_{\mu \nu}$ as

$$
T_{(\mathrm{BY})}^{\mu \nu}=\frac{1}{8 \pi G}\left(\gamma^{\mu \nu} K-K^{\mu \nu}\right),
$$

and $T_{(\mathrm{ct})}^{\mu \nu}$ is the terms from the counter terms. By using appropriate counter terms, the stress-energy tensor becomes finite at the boundary $r \rightarrow \infty$ and we define

$$
\widetilde{T}_{\nu}^{\mu} \equiv \lim _{r \rightarrow \infty} r^{z+3-4 \theta / 3}\left(\hat{S}_{\nu}^{0} \hat{v}^{\mu}-\hat{S}_{\nu}^{a} e_{a}^{\mu}\right)
$$

and the current is given by

$$
J^{\mu} \equiv \lim _{r \rightarrow \infty} r^{z+3-4 \theta / 3}\left(\hat{J}^{0} \hat{v}^{\mu}+\hat{J}^{a} e_{a}^{\mu}\right)
$$

\footnotetext{
${ }^{9}$ Even though $\hat{A}_{0}$ starts from $\mathcal{O}\left(r^{z+3-\theta}\right)$, the leading contribution to the effective action is $\hat{J}^{0} \mathcal{A}_{0}$ and hence, the regular term in $\hat{J}^{0}$ originates at the same order as the others, namely $\mathcal{O}\left(r^{-z-3+4 \theta / 3}\right)$.
} 
As we will see later, it is also different from the ordinary stress-energy tensor on the boundary but contains the contributions from the external gauge field.

Now, we calculate the renormalized stress-energy tensor from the first order solution (3.24). In order to regularize the stress-energy tensor, and also other expectation values, we introduce the following counter terms;

$$
S_{\mathrm{ct}}=\frac{1}{16 \pi G} \int d^{4} x \sqrt{-\gamma}\left[-(5+z-2 \theta) e^{-\frac{1}{2} \nu \phi}+\frac{z+3-\theta}{2} e^{\left(\lambda-\frac{1}{2} \nu\right) \phi} \gamma^{\mu \nu} A_{\mu} A_{\nu}\right] .
$$

Although this counter term is apparently not gauge invariant, the effective action is still invariant under boundary gauge transformations (up to boundary terms on the boundary). The second term in (4.15) is expanded as

$$
\sqrt{-\gamma} e^{\left(\lambda-\frac{1}{2} \nu\right) \phi} \gamma^{\mu \nu} A_{\mu} A_{\nu} \propto a^{\frac{\theta}{3(3-\theta)}} r^{z+3-4 \theta / 3}-a^{\frac{\theta}{3(3-\theta)}} r_{0}^{z+3-\theta}+\frac{2}{a}\left(\mathcal{A}_{t}+v^{i} \mathcal{A}_{i}\right) \cdots
$$

Near the boundary, $r \rightarrow \infty$, the first term is leading and will cancel with the divergent part in the original action. The second and third terms give finite contributions to the effective action. The ellipsis denotes terms that are vanishing as we take the cutoff surface to the boundary. We therefore observe that indeed the finite terms are not gauge invariant but are linear in the gauge field.

Under the boundary gauge transformation $\delta \mathcal{A}=d \Lambda$, the finite term in (4.15) transforms as

$$
\delta_{\Lambda} S_{\mathrm{ct}} \propto \int d^{4} x\left[\partial_{t} \frac{\Lambda}{a}+\partial_{i} \frac{\Lambda v^{i}}{a}\right]
$$

where we used (3.36). Therefore the transformed terms are surface terms and should vanish at infinity on the boundary. The coefficient of these terms is indeed the conserved current (see equation (4.23)) and (4.23) can be written as

$$
\delta_{\Lambda} S_{\mathrm{ct}} \propto \int d^{4} x J^{\mu} \partial_{\mu} \Lambda=\int d^{4} x\left(\partial_{\mu} J^{\mu}\right) \Lambda=0
$$

Therefore if no charge is leaking to infinity on the boundary the effective action is gauge invariant.

By using the counter terms (4.15), the renormalized stress-energy tensor is calculated as

$$
\begin{aligned}
& \widetilde{T}_{0}^{0}=\frac{1}{8 \pi G}\left(-\frac{3-\theta}{2} a^{\frac{\theta}{3(3-\theta)}} r_{0}^{z+3-\theta}-\frac{z-1}{a} v^{i} \mathcal{A}_{i}\right) \\
& \widetilde{T}^{i}{ }_{0}=\frac{1}{8 \pi G}\left[-\frac{z+3-\theta}{2} a^{\frac{\theta}{3(3-\theta)}} r_{0}^{z+3-\theta} v^{i}+\frac{z-1}{a} v^{i} \mathcal{A}_{t}+\frac{1}{2} r_{0}^{3-\theta} \sigma_{i j} v^{j}\right. \\
& \left.\quad+\frac{z(z+3-\theta)}{4(z-1)} r_{0}^{2 z-\theta}\left(\partial_{i} r_{0}+\frac{\theta}{3(3-\theta) z} \frac{r_{0}}{a} \partial_{i} a\right)\right] \\
& \widetilde{T}^{0}{ }_{i}=\frac{1}{8 \pi G} \frac{z-1}{a} \mathcal{A}_{i}, \\
& \widetilde{T}^{i}{ }_{j}=\frac{1}{8 \pi G}\left[\frac{z}{2} a^{\frac{\theta}{3(3-\theta)}} r_{0}^{z+3-\theta} \delta_{i j}-\frac{1}{2} r_{0}^{3-\theta} \sigma_{i j}+\frac{z-1}{a} v^{i} \mathcal{A}_{j}-\frac{z-1}{a}\left(\mathcal{A}_{t}+v^{k} \mathcal{A}_{k}\right) \delta_{i j}\right] .
\end{aligned}
$$


The current $J^{\mu}$ is obtained as

$$
J^{0}=\frac{z-1}{16 \pi G a} \quad, \quad J^{i}=\frac{z-1}{16 \pi G a} v^{i} .
$$

The expectation value of the dual operator of the dilaton $\phi$ is also calculated as

$$
\begin{aligned}
\left\langle\mathcal{O}_{\phi}\right\rangle= & -\frac{1}{16 \pi G} \frac{9(z-1)+(3-\theta) \theta}{\sqrt{6(3-\theta)[3(z-1)-\theta]}} a^{\frac{\theta}{3(3-\theta)}} r_{0}^{z+3-\theta} \\
& +\frac{1}{16 \pi G} \frac{12(z-1)(6-\theta)}{\sqrt{6(3-\theta)[3(z-1)-\theta]}} \frac{1}{a}\left(\mathcal{A}_{t}+v^{i} \mathcal{A}_{i}\right)
\end{aligned}
$$

We also calculate the entropy current that is given by [11]

$$
J_{S}^{\mu}=\frac{\sqrt{h}}{4 G} \frac{n^{\mu}}{n^{0}}
$$

where $\sqrt{h}$ is the volume form on the time-slice at the horizon and $n^{\mu}$ is the normal vector to the horizon. By using the first order solution (3.24), the entropy current becomes

$$
\begin{aligned}
& J_{S}^{0}=\frac{1}{4 G} r_{0}^{3-\theta}, \\
& J_{S}^{i}=\frac{1}{4 G} r_{0}^{3-\theta} v^{i}-\frac{1}{8(z-1) G} a^{-\frac{\theta}{3(3-\theta)}} r_{0}^{z-\theta}\left(z \partial_{i} r_{0}+\frac{\theta}{3(3-\theta)} \frac{r_{0}}{a} \partial_{i} a\right) .
\end{aligned}
$$

\section{Hyperscaling-violating Lifshitz hydrodynamics}

\subsection{Thermodynamics}

In order to consider the relation between the stress-energy tensor (4.13) and that for fluids, we first calculate the thermodynamic functions. The energy $E$, entropy $S$ and charge $N$ in volume $V$ (in the $x^{i}$ directions) of the Lifshitz black hole geometry are given by

$$
\begin{aligned}
E & =\mathcal{E} V=\frac{d-1-\theta}{16 \pi G} a^{\frac{\theta}{(d-1)(d-1-\theta)}} r_{0}^{z+d-1-\theta} V, \\
S & =s V=\frac{1}{4 G} r_{0}^{d-1-\theta} V \\
N & =n V=\frac{z-1}{16 \pi G a} V
\end{aligned}
$$

where we have also defined $\mathcal{E}, s$ and $n$, which are densities of energy, entropy and charge. Here we have also taken into account the effect of the coordinate redefinition (3.10), and hence the mass has an additional factor of $a^{\frac{\theta}{(d-1)(d-1-\theta)}}$.

Now, we consider the first law of thermodynamics;

$$
d E=T d S-P d V+\mu d N
$$


where the variations with respect to entropy $S$, volume $V$ and charge $N$ give temperature $T$, pressure $P$ and chemical potential $\mu$, which are calculated as

$$
\begin{aligned}
& T=\left(\frac{\partial E}{\partial S}\right)_{V, N}=\frac{z+d-1-\theta}{4 \pi} a^{\frac{\theta}{(d-1)(d-1-\theta)}} r_{0}^{z}, \\
& P=-\left(\frac{\partial E}{\partial V}\right)_{S, N}=\frac{1}{16 \pi G}\left(z-\frac{\theta}{d-1}\right) a^{\frac{\theta}{(d-1)(d-1-\theta)}} r_{0}^{z+d-1-\theta}, \\
& \mu=\left(\frac{\partial E}{\partial N}\right)_{S, V}=-\frac{\theta}{(d-1)(z-1)} a^{1+\frac{\theta}{(d-1)(d-1-\theta)}} r_{0}^{z+d-1-\theta} .
\end{aligned}
$$

For $d=4$, the energy density, pressure, entropy density, charge density and chemical potential are given by

$$
\begin{array}{rlrl}
\mathcal{E} & =\frac{3-\theta}{16 \pi G} a^{\frac{\theta}{3(3-\theta)}} r_{0}^{z+3-\theta}, & P & =\frac{1}{16 \pi G}\left(z-\frac{\theta}{d-1}\right) a^{\frac{\theta}{3(3-\theta)}} r_{0}^{z+3-\theta}, \\
s & =\frac{1}{4 G} r_{0}^{3-\theta}, & n & =\frac{z-1}{16 \pi G} a^{-1}, \\
\mu & =-\frac{\theta}{3(z-1)} a^{1+\frac{\theta}{3(3-\theta)}} r_{0}^{z+3-\theta} . &
\end{array}
$$

Note that at $\theta=0$, the chemical potential $\mu$ vanishes.

\subsection{Relation to hydrodynamics in Newton-Cartan theory}

In this section we will rewrite the boundary stress tensor in terms of the Newton-Cartan geometry data along the lines of [54]-[56] following the detailed formalism of [1].

In the Newton-Cartan theory, (that is explained in more detail in appendix B), the spacelike vielbein, inverse timelike vielbein $\bar{v}^{\mu}$ and $\bar{e}_{\mu}^{a}$ transform under the Milne boost. In this paper, $\bar{v}^{\mu}$ and $\bar{e}_{\mu}^{a}$ indicate the vielbeins in an arbitrary frame.

There is a special frame, the "holographic frame," introduced in [1], where the vielbeins are given by

$$
\bar{v}^{\mu}=\hat{v}^{\mu} \quad, \quad \bar{e}_{\mu}^{a}=\hat{e}_{\mu}^{a} .
$$

where $\hat{v}^{\mu}$ and $\hat{e}_{\mu}^{a}$ are defined on the gravity side, in (4.6), and $\hat{v}^{\mu}$ always satisfies in any frame

$$
\hat{v}^{\mu}=u^{\mu} \equiv(1, \vec{v}),
$$

where $u^{\mu}$ is the fluid velocity. The timelike vielbein and inverse spacelike vielbein do not transform under the Milne boost and hence are simply referred to as $\tau_{\mu}$ and $e_{a}^{\mu}$, respectively.

The renormalized boundary stress-energy tensor, which we have calculated in the previous section, can be expressed in the following form;

$$
\widetilde{T}^{\mu}{ }_{\nu}=-\mathcal{E} \hat{v}^{\mu} \tau_{\nu}+(P-n \mu) \hat{h}^{\mu}{ }_{\nu}-\kappa \tau_{\nu} h^{\mu \rho} \partial_{\rho} T-\eta \sigma_{a b} e_{a}^{\mu} \hat{e}_{\nu}^{b}+n \hat{v}^{\mu} \mathcal{A}_{\nu}-n \hat{v}^{\rho} \mathcal{A}_{\rho} \delta^{\mu}{ }_{\nu}
$$

where the spatial metric is defined in terms of the vielbeins $e_{a}^{\mu}$ and $\hat{e}_{\mu}^{a}$;

$$
h^{\mu \nu}=e_{a}^{\mu} e_{a}^{\nu}, \quad \hat{h}^{\mu}{ }_{\nu}=e_{a}^{\mu} \hat{e}_{\nu}^{a} .
$$


The energy density $\mathcal{E}$, pressure $P$, particle number density $n$ and chemical potential $\mu$ are given by (5.8). The transport coefficients - heat conductivity $\kappa$, shear viscosity $\eta$ and bulk viscosity $\zeta$ are also read off as

$$
\kappa=\frac{1}{8(z-1) G} a^{-\frac{\theta}{3(3-\theta)}} r_{0}^{z+1-\theta}, \quad \eta=\frac{1}{16 \pi G} r_{0}^{3-\theta}, \quad \zeta=0,
$$

where the bulk viscosity $\zeta$ is the coefficient of the expansion $\partial_{i} v^{i}$ in the stress-energy tensor (5.11). Hence, $\zeta=0$ can be deduced from the absence of $\partial_{i} v^{i}$ in (5.11). The stressenergy tensor can also be expressed in terms of the energy flow $\widetilde{\mathcal{E}}^{\mu}$, momentum density $\widetilde{\mathcal{P}}_{\mu}$, stress tensor $\widetilde{\mathcal{T}}^{\mu}{ }_{\nu}$ and current $J^{\mu}$, which are defined by

$$
\begin{aligned}
\widetilde{\mathcal{E}}^{\mu} & =-\widetilde{T}_{0}^{\mu}{ }_{\nu} \hat{v}^{\nu} \\
\widetilde{\mathcal{P}}_{\nu} & =\widetilde{T}_{0}^{\mu}{ } \tau_{\mu} \hat{h}^{\rho}{ }_{\nu} \\
\widetilde{\mathcal{T}}^{\mu}{ }_{\nu} & =\widetilde{T}_{0}^{\rho}{ }_{0} \hat{h}^{\mu}{ }_{\rho} \hat{h}^{\sigma}{ }_{\nu}
\end{aligned}
$$

where

$$
\widetilde{T}_{0 \nu}^{\mu}=\widetilde{T}^{\mu}{ }_{\nu}+n \mu \hat{h}^{\mu}{ }_{\nu}-J^{\mu} \mathcal{A}_{\nu}+J^{\rho} \mathcal{A}_{\rho} \delta^{\mu}{ }_{\nu} .
$$

Then, $\widetilde{T}^{\mu}{ }_{\nu}$ is written as

$$
\widetilde{T}^{\mu}{ }_{\nu}=-\widetilde{\mathcal{E}}^{\mu} \tau_{\nu}+\hat{v}^{\mu} \widetilde{\mathcal{P}}_{\nu}+\widetilde{\mathcal{T}}^{\mu}{ }_{\nu}-n \mu \hat{h}^{\mu}{ }_{\nu}+J^{\mu} \mathcal{A}_{\nu}-J^{\rho} \mathcal{A}_{\rho} \delta^{\mu}{ }_{\nu}
$$

Comparing (5.18) with (5.11), we obtain

$$
\begin{aligned}
\widetilde{\mathcal{E}}^{\mu} & =\mathcal{E} \hat{v}^{\mu}-\kappa h^{\mu \rho} \partial_{\rho} T \\
\widetilde{\mathcal{P}}_{\nu} & =0 \\
\widetilde{\mathcal{T}}^{\mu}{ }_{\nu} & =P \hat{h}^{\mu}{ }_{\nu}-\eta \sigma_{a b} e_{a}^{\mu} \hat{e}^{b} \\
J^{\mu} & =n \hat{v}^{\mu}
\end{aligned}
$$

The above stress-energy tensor can be identified with that in the Newton-Cartan theory. ${ }^{10}$

In the Newton-Cartan theory, the geometry is described by the timelike vielbein $\tau_{\mu}$, spatial (inverse) metric $h^{\mu \nu}$, timelike inverse vielbein $\bar{v}^{\mu}$ and gauge field $B_{\mu}$. Here, the spacelike vielbein, its inverse and spatial metric with lower indices are denoted as $\bar{e}_{\mu}^{a}, e_{a}^{\mu}$ and $\bar{h}_{\mu \nu}$. The energy current $\mathcal{E}^{\mu}$, stress tensor $\mathcal{T}_{\mu \nu}$, momentum density $\mathcal{P}_{\mu}$ and mass current $\mathcal{J}^{\mu}$ are the conserved quantities associated to $\tau_{\mu}, h^{\mu \nu}, \bar{v}^{\mu}$ and $B_{\mu}$, respectively. For a generic fluid in Eckart frame, they are given by [70]

$$
\begin{aligned}
\mathcal{E}^{\mu} & =\mathcal{E} u^{\mu}+\frac{1}{2} \rho u^{2} u^{\mu}-\kappa h^{\mu \nu} \partial_{\nu} T+h^{\mu \rho} u^{\sigma} \mathcal{T}_{\rho \sigma}, \\
\mathcal{P}_{\mu} & =\rho u_{\mu}, \\
\mathcal{T}_{\mu \nu} & =P \bar{h}_{\mu \nu}+\rho u_{\mu} u_{\nu}-\eta \sigma_{\rho \sigma} \bar{P}_{\mu}^{\rho} \bar{P}_{\nu}^{\sigma}-\zeta \vartheta \bar{h}_{\mu \nu}, \\
\mathcal{J}^{\mu} & =\rho u^{\mu}
\end{aligned}
$$

\footnotetext{
${ }^{10}$ There can be ambiguities in the definition of the stress tensor. In relativistic CFTs they are known to affect higher-order transport coefficients, [75]. In non-relativistic scaling theories the situation is even more sensitive as they affect even the idea hydrodynamics equations as we will see further. Moreover, as we discuss in section 6 , they also affect the bulk viscosity.
} 
where

$$
\bar{P}_{\nu}^{\mu} \equiv e_{a}^{\mu} \bar{e}_{\nu}^{a} \quad, \quad \vartheta \equiv \partial_{i} v^{i}
$$

$\bar{P}_{\nu}^{\mu}$ is the projector to the spatial directions and $\vartheta$ is the expansion of the fluid.

The fluid velocity $u^{\mu}$ satisfies the normalization condition $\tau_{\mu} u^{\mu}=1$, and is given by $u^{\mu}=\left(1, u^{i}\right)$ for $\tau=d t$.

In the Newton-Cartan theory, the generic stress-energy tensor (not only for the fluid above) is defined from the energy current $\mathcal{E}^{\mu}$, stress tensor $\mathcal{T}_{\mu \nu}$, and momentum density $\mathcal{P}_{\mu}$ as $^{11}$

$$
\bar{T}^{\mu}{ }_{\nu}=-\mathcal{E}^{\mu} \tau_{\nu}+\bar{v}^{\mu} \mathcal{P}_{\nu}+h^{\mu \rho} \mathcal{T}_{\rho \nu} .
$$

The Newton-Cartan theory has a symmetry which is known as Milne boost; ${ }^{12}$

$$
\begin{aligned}
\bar{v}^{\mu} \rightarrow \bar{v}^{\prime \mu} & =\bar{v}^{\mu}+h^{\mu \nu} V_{\nu}, \\
B \rightarrow B^{\prime} & =B+\bar{P}_{\mu}^{\nu} V_{\nu} d x^{\mu}-\frac{1}{2} h^{\mu \nu} V_{\mu} V_{\nu} \tau_{\rho} d x^{\rho} .
\end{aligned}
$$

and we can introduce the Milne boost invariant combination for the gauge field 1-form as $[56,70]$

$$
\widehat{B}=B+u_{\mu} d x^{\mu}-\frac{1}{2} u^{2} \tau_{\rho} d x^{\rho}
$$

where

$$
u_{\mu}=\bar{h}_{\mu \nu} u^{\nu}, \quad u^{2}=\bar{h}_{\mu \nu} u^{\mu} u^{\nu}
$$

We also define a Milne boost invariant stress-energy tensor as

$$
T^{\mu}{ }_{\nu}=\bar{T}^{\mu}{ }_{\nu}+\mathcal{J}^{\mu} B_{\nu}-\mathcal{J}^{\mu} \widehat{B}_{\nu}=\bar{T}^{\mu}{ }_{\nu}-\mathcal{J}^{\mu}\left(u_{\nu}-\frac{1}{2} \tau_{\nu} u^{2}\right) .
$$

We choose $\bar{v}^{\mu}=u^{\mu}$ and we have

$$
\begin{aligned}
& u_{\mu}=\bar{h}_{\mu \nu} u^{\nu}=\bar{h}_{\mu \nu} \bar{v}^{\mu}=0, \\
& u^{2}=\bar{h}_{\mu \nu} u^{\mu} u^{\nu}=0,
\end{aligned}
$$

and then, (5.31) and (5.33) give

$$
B=\widehat{B}, \quad T_{\nu}^{\mu}=\bar{T}^{\mu}{ }_{\nu} .
$$

Therefore, the Milne boost invariants $\widehat{B}$ and $T^{\mu}{ }_{\nu}$ are the same as $B$ and $\bar{T}^{\mu}{ }_{\nu}$ in $\bar{v}^{\mu}=$ $u^{\mu}$ frame.

\footnotetext{
${ }^{11}$ We always denote stress-energy tensors by the letter $T$ and their spatial projection (the stress tensor) by the letter $\mathcal{T}$.

${ }^{12}$ When the geometry is torsion-free then gauge invariance and Milne boost invariance can be simultaneously present. However in more general cases they are incompatible, [54]-[56]. In the case described in this paper the geometry is torsion-free.
} 
We further define the Milne boost invariants for the energy flow $\widehat{\mathcal{E}}^{\mu}$, momentum density $\widehat{\mathcal{P}}_{\mu}$, stress tensor $\widehat{\mathcal{T}}^{\mu}{ }_{\nu}$ from the Milne boost-invariant stress-energy tensor $T^{\mu}{ }_{\nu}$ as

$$
\begin{aligned}
\widehat{\mathcal{E}}^{\mu} & =-T^{\mu}{ }_{\nu} u^{\nu}, \\
\widehat{\mathcal{P}}_{\nu} & =T^{\mu}{ }_{\rho} \tau_{\mu} \bar{P}_{\nu}^{\rho}, \\
\widehat{\mathcal{T}}^{\mu}{ }_{\nu} & =T^{\rho}{ }_{\sigma} \bar{P}_{\rho}^{\mu} \bar{P}_{\nu}^{\sigma},
\end{aligned}
$$

and then, $T^{\mu}{ }_{\nu}$ is written as

$$
T^{\mu}{ }_{\nu} \equiv-\widehat{\mathcal{E}}^{\mu} \tau_{\nu}+u^{\mu} \widehat{\mathcal{P}}_{\nu}+\widehat{\mathcal{T}}^{\mu}{ }_{\nu}
$$

From (5.23)-(5.25), (5.28), (5.33) and (5.40), the Milne boost invariants are expressed as

$$
\begin{aligned}
\widehat{\mathcal{E}}^{\mu} & =\mathcal{E} u^{\mu}-\kappa h^{\mu \nu} \partial_{\nu} T \\
\widehat{\mathcal{P}}_{\mu} & =0 \\
\widehat{\mathcal{T}}_{\mu \nu} & =P \bar{h}_{\mu \nu}-\eta \sigma_{\rho \sigma} \bar{P}_{\mu}^{\rho} \bar{P}_{\nu}^{\sigma}-\zeta \theta \bar{h}_{\mu \nu},
\end{aligned}
$$

and here $\bar{h}^{\mu \nu}$ and $\bar{P}_{\nu}^{\mu}$ are given by those in $\bar{v}^{\mu}=u^{\mu}$ frame. Then, (5.23)-(5.25) take the same form to (5.41)-(5.43), respectively, for $v^{i}=u^{i}$ and $\zeta=0$. Therefore, we identify the energy flow $\widetilde{\mathcal{E}}^{\mu}$, momentum density $\widetilde{\mathcal{P}}_{\mu}$ and stress tensor $\widetilde{\mathcal{T}}^{\mu}{ }_{\nu}$, which are calculated from the black hole geometry in the previous section, with the Milne boost-invariants $\widehat{\mathcal{E}}^{\mu}, \widehat{\mathcal{P}}_{\mu}$ and $\widehat{\mathcal{T}}^{\mu}{ }_{\nu}$, respectively. We also identify the gauge field $\mathcal{A}_{\mu}$, which comes from the constant mode of the bulk gauge field $A_{\mu}$, with the Milne boost-invariant combination $\widehat{B}_{\mu}$ as

$$
\mathcal{A}_{\mu}=m \widehat{B}_{\mu}-\mu \tau_{\mu}
$$

where $m$ is the coupling constant for the gauge field $B_{\mu}$, which is equivalently in the nonrelativistic language the mass per particle. Then, the mass current $\mathcal{J}^{\mu}$ is related to the particle number current $J^{\mu}$ as

$$
\mathcal{J}^{\mu}=m J^{\mu}
$$

It is then straightforward to verify that the stress-energy tensor $\widetilde{T}^{\mu}{ }_{\nu}$, which we calculated from the black hole geometry, is related to the Milne boost-invariant stress-energy tensor $T^{\mu}{ }_{\nu}$ in the Newton-Cartan theory as

$$
\widetilde{T}^{\mu}{ }_{\nu}=T^{\mu}{ }_{\nu}+\mathcal{J}^{\mu} \widehat{B}_{\nu}-\mathcal{J}^{\rho} \widehat{B}_{\rho} \delta^{\mu}{ }_{\nu} .
$$

The conservation law for these Milne boost-invariants is given by [71]

$$
\begin{aligned}
D_{\mu} \widehat{\mathcal{E}}^{\mu} & =\hat{v}^{\mu} \widehat{H}_{\mu \nu} \mathcal{J}^{\nu}-\frac{1}{2}\left(h^{\mu \rho} D_{\rho} \hat{v}^{\nu}+h^{\nu \rho} D_{\rho} \hat{v}^{\mu}\right) \widehat{\mathcal{T}}_{\mu \nu}, \\
h^{\rho \mu} h^{\sigma \nu} D_{\rho} \widehat{\mathcal{T}}_{\mu \nu} & =h^{\sigma \nu}\left[\hat{v}^{\mu} D_{\nu} \widehat{\mathcal{P}}_{\mu}-D_{\mu}\left(\hat{v}^{\mu} \widehat{\mathcal{P}}_{\nu}\right)+\widehat{H}_{\mu \nu} \mathcal{J}^{\mu}\right]
\end{aligned}
$$

where $\widehat{H}=d \widehat{B}$. The covariant derivative $D_{\mu}$ is defined as usual in the Newton-Cartan geometry (see appendix B). 
In terms of the Milne boost invariant stress-energy tensor, the conservation law can be expressed as

$$
D_{\mu} T^{\mu}{ }_{\nu}=J^{\mu} \mathcal{F}_{\mu \nu}=\mathcal{J}^{\mu} \widehat{H}_{\mu \nu}
$$

since $\widehat{\mathcal{P}}_{\mu}=0$. More details are given in appendix B.

The renormalized stress-energy tensor $\widetilde{T}^{\mu}{ }_{\nu}$ satisfies the conservation equations which are equivalent to (5.47) and (5.48). These equations come from the following components of the bulk equations of motion;

$$
\begin{aligned}
n^{\mu} \gamma^{\nu \rho} R_{\mu \nu} & =8 \pi G n^{\mu} \gamma^{\nu \rho} T_{\mu \nu}^{(\text {bulk })}, \\
n_{\nu} \nabla_{\mu}\left(e^{\lambda \phi} F^{\mu \nu}\right) & =0
\end{aligned}
$$

where $n_{\mu}$ and $\gamma_{\mu \nu}$ are the normal vector and the induced metric on the boundary, respectively. In order to derive the conservation equations for the first order fluids, we have to calculate the constraint equations (5.50), (5.51) to second order in the derivative expansion. The correction terms at each order do not contribute to the constraint equations at that same order, and hence, we do not need to solve the differential equation for the correction terms to second order.

The constraint equations (5.50), (5.51) can be expressed in terms of the fluid variables $\mathcal{E}, P, n$ and $v^{i}$ defined in (5.23)-(5.26) as

$$
\begin{aligned}
0= & \partial_{t} \mathcal{E}+v^{i} \partial_{i} \mathcal{E}+(\mathcal{E}+P) \partial_{i} v^{i} \\
& -\frac{1}{2} \eta \sigma_{i j} \sigma_{i j}-\partial_{i}\left(\kappa \partial_{i} T\right) \\
0= & \partial_{i} P-n \partial_{i} \mu+n \mathcal{F}_{t i}+n v^{j} \mathcal{F}_{j i}-\partial_{j}\left(\eta \sigma_{i j}\right), \\
0= & \partial_{t} n+\partial_{j}\left(n v^{j}\right) .
\end{aligned}
$$

Note that the main difference of these equation in the hyperscaling violating case studied here and the case with $\theta=0$ studied in [1] is the appearance of the term $n \partial_{i} \mu$ in the Navier-Stokes-like equation (5.53). Here we have a chemical potential in the absence of an external Newtonian potential and this is due to hyperscaling violation.

The fluid equations (5.52)-(5.54) are equations for energy density $\mathcal{E}$, pressure $P$, velocity field $v^{i}$, particle number density $n$ and temperature $T$. There are also constituency relations between these variables. In particular, the temperature and energy density are related to each other, and hence are not independent. For the fluid dual to the Lifshitz geometry, the energy density is constrained first by the Lifshitz Ward identity with hyperscaling violation. This is given by

$$
\left(z-\frac{\theta}{d-1}\right) \mathcal{E}=(d-1-\theta) P .
$$

The transport coefficients $\eta$ and $\kappa$ are not constant but depend on the temperature, and also on the particle number density in this case, as in (5.13). By using (5.31) and (5.44), the gauge field $\mathcal{A}$ is rewritten in terms of the velocity field $v^{i}$ and the external gauge field 
of $B$;

$$
\begin{aligned}
\mathcal{A} & =m\left(B+u_{\mu} d x^{\mu}-\frac{1}{2} u^{2} \tau_{\rho} d x^{\rho}\right)-\mu \tau \\
& =m\left(B+v^{i} d x^{i}-\frac{1}{2} \vec{v}^{2} d t\right)-\mu d t
\end{aligned}
$$

Therefore, we have 5 equations for 5 independent variables $\mathcal{E}, v^{i}$ and $n$.

We also have external sources in the hydrodynamic equations: one is of course the space metric that is flat here (but in subsequent sections this will change). The other is the external gauge field $B_{\mu}$ that couples to the U(1) current. In ordinary non-relativistic hydrodynamics, $B_{t}$ would be the ordinary Newtonian potential that couples to mass $[1,54-56]$.

\subsection{The entropy current}

The entropy current (4.25) can be expressed as

$$
J_{S}^{\mu}=s \hat{v}^{\mu}-\frac{\kappa}{T} h^{\mu \rho} \partial_{\rho} T
$$

where the entropy density $s$ is given by

$$
s=\frac{1}{4 G} r_{0}^{3-\theta} .
$$

The entropy current satisfies the following relation with the (internal) energy density $\widehat{\mathcal{E}}$ and pressure $P$;

$$
T J_{S}^{\mu}=\widehat{\mathcal{E}}^{\mu}+P \hat{v}^{\mu}-\mu J^{\mu}=-T^{\mu}{ }_{\nu} \hat{v}^{\nu}+(P-\mu n) \hat{v}^{\mu} .
$$

It also satisfies the second law. By using (5.52), the divergence of $J_{S}^{\mu}$ is expressed as

$$
\partial_{\mu} J_{S}^{\mu}=\frac{1}{2} \frac{\eta}{T} \sigma_{i j} \sigma_{i j}+\frac{\kappa}{T^{2}}\left(\partial_{i} T\right)^{2},
$$

which is manifestly non-negative. Therefore, the entropy current satisfies the second law. The entropy density $s=J_{S}^{0}$ saturates the KSS bound [69];

$$
\frac{\eta}{s}=\frac{1}{4 \pi}
$$

\section{The relation to dimensional reduction}

In the previous section, we studied the fluids in the field theory dual of the Lifshitz spacetime with hyperscaling-violation. We found that the fluid has zero bulk viscosity $\zeta=0$.

It is known that the Lifshitz space-time with hyperscaling-violation can be obtained by the dimensional reduction from the higher dimensional Lifshitz space-time without hyperscaling-violation, [18]. As discussed in [1], the fluid for the Lifshitz space-time without hyperscaling-violation also has zero bulk viscosity. Upon compactification, this Lifshitz geometry with flat internal space and constant radius, becomes the lower dimensional 
geometry with hyperscaling violation. In such a case it was shown, [72, 73], that the bulk viscosity becomes non-zero after dimensional reduction.

In this section, we will first derive the general fluid equations in the higher-dimensional theory by allowing also the internal volume to be an additional thermodynamic variable. Then we will reduce to the lower dimension. We will show that the appropriate reduction that corresponds to the thermodynamic ansatz in the lower dimension is compatible with our four-dimensional results of the previous section.

We first consider the following theory, which is the Einstein gravity with the Maxwell field and a single scalar field;

$$
S=\frac{1}{16 \pi G} \int d^{D+1} x \sqrt{-g_{D}}\left(R-2 \Lambda-\frac{1}{2}\left(\partial \phi_{1}\right)^{2}-\frac{1}{4} e^{\tilde{\lambda} \phi_{1}} F^{2}\right),
$$

where $D=d-\theta$, and we assumed that $\theta$ is a negative integer at this moment. This model has the Lifshitz space-time without hyperscaling-violation as a solution;

$$
d s_{D}^{2}=-r^{2 z} d t^{2}+\frac{d r^{2}}{r^{2}}+\sum_{i=1}^{d-\theta-1} r^{2}\left(d x^{i}\right)^{2},
$$

with the following gauge field and dilaton;

$$
A_{t}=a \sqrt{\mu} r^{z+d-\theta-1}, \quad \quad e^{\tilde{\lambda} \phi_{1}}=a^{-2} r^{-2(d-\theta-1)},
$$

where the parameters $z, a$ and $\mu$ are related to the parameters of the action (coupling constants) as

$$
\begin{aligned}
\tilde{\lambda}^{2} & =2 \frac{d-\theta-1}{z-1}, \\
\Lambda & =-\frac{(z+d-\theta-1)(z+d-\theta-2)}{2}, \\
\mu & =\frac{2(z-1)}{z+d-\theta-1} .
\end{aligned}
$$

We compactify the $(-\theta)$-dimensional extra dimensions and consider the dimensional reduction to $(d+1)$-dimensional spacetime. The metric is decomposed as

$$
d s_{D}^{2}=e^{-\tilde{\nu} \phi_{2}} d s^{2}+\sum_{i=d}^{d-\theta-1} e^{-(d-1) \tilde{\nu} \phi_{2} / \theta}\left(d x^{i}\right)^{2},
$$

where $d s^{2}$ is the metric after the dimensional reduction and we compactified $x^{i}$-directions with $i=d, \cdots, d-\theta-1$ to circles with period $x^{i} \sim x^{i}+1$. The constant $\tilde{\nu}$ is given by

$$
\tilde{\nu}^{2}=-\frac{2 \theta}{(d-1)(d-\theta-1)} .
$$

Then, after the dimensional reduction, the action becomes

$$
S=\frac{1}{16 \pi G} \int d^{d+1} x \sqrt{-g}\left(R-2 \Lambda e^{-\tilde{\nu} \phi_{2}}-\frac{1}{2}\left(\partial \phi_{1}\right)^{2}-\frac{1}{2}\left(\partial \phi_{2}\right)^{2}-\frac{1}{4} e^{\tilde{\lambda} \phi_{1}+\tilde{\nu} \phi_{2}} F^{2}\right),
$$


Now, the geometry has hyperscaling-violation because of the redefinition of the metric, and given by

$$
\begin{aligned}
& d s^{2}=r^{-2 \theta /(d-1)} d \tilde{s}^{2} \\
& d \tilde{s}^{2}=-r^{2 z} d t^{2}+\frac{d r^{2}}{r^{2}}+\sum_{i=1}^{d-1} r^{2}\left(d x^{i}\right)^{2} .
\end{aligned}
$$

while the additional dilaton field $\phi_{2}$ is

$$
e^{\tilde{\nu} \phi_{2}}=r^{-2 \theta /(d-1)} .
$$

More generally, $\phi_{2}$ also has the constant mode as is $\phi_{1}$ but it is independent from the gauge field. Then, the solution becomes

$$
\begin{aligned}
d s^{2} & =e^{2 \chi} d \tilde{s}^{2} \\
d \tilde{s}^{2} & =-r^{2 z} d t^{2}+\frac{d r^{2}}{r^{2}}+\sum_{i=1}^{d-1} r^{2}\left(d x^{i}\right)^{2}, \\
e^{\tilde{\nu} \phi_{2}} & =e^{2 \chi}=e^{2 \chi_{0}} r^{-2 \theta /(d-1)}
\end{aligned}
$$

where $\chi_{0}$ is an arbitrary constant. We define a new parameter $b$ as

$$
\chi_{0}=-\frac{\theta}{(d-1)(d-1-\theta)} \log b .
$$

In a sense $b$ parametrizes the internal volume.

Now, the model has 2 dilatons instead of one. This solution has an additional parameter $b$ which is the constant mode of the additional dilaton $\phi_{2}$. The solution becomes equivalent to that in the previous sections if the dilatons satisfy the following condition;

$$
\lambda \phi=\left(1+\frac{\theta}{(d-1)(d-\theta-1)}\right) \tilde{\lambda} \phi_{1}=\left(1+\frac{(d-1)(d-\theta-1)}{\theta}\right) \tilde{\nu} \phi_{2} .
$$

In fact the above solution satisfies this condition except for the constant mode $b$, and hence, it is equivalent to the solution in the previous sections if

$$
b=a .
$$

As already noted, the value of $b$ controls the volume of the internal dimensions. For the higher-dimensional Lifshitz geometry (6.2), we have

$$
b=1
$$

but arbitrary $b$ can be introduced by the redefinition of the coordinates in the extra dimensions.

The solution which describes the hydrodynamics can be calculated in a similar fashion to the previous sections. We now specialize to $d=4$. The first order solution of the 
derivative expansion is obtained as

$$
\begin{aligned}
d s_{D}^{2}= & d \tilde{s}^{2}+\sum_{i=3}^{3-\theta} e^{-(d-1) \tilde{\nu} \phi_{2} / \theta}\left(d x^{i}\right)^{2} \\
d s^{2}= & r^{-2 \theta /(d-1)} e^{\nu \varphi_{2}} d \tilde{s}^{2} \\
d \tilde{s}^{2}= & -r^{2 z} f d t^{2}+2 b^{-\frac{\theta}{3(3-\theta)}} r^{z-1} d t d r+r^{2}\left(d x^{i}-v^{i} d t\right)^{2} \\
& +\frac{2}{3-\theta} b^{-\frac{\theta}{3(3-\theta)}} r^{z} \partial_{i} v^{i} d t^{2}-r^{2} F_{1}(r) \sigma_{i j}\left(d x^{i}-v^{i} d t\right)\left(d x^{j}-v^{j} d t\right) \\
& -\frac{2 \theta}{3(3-\theta)} F_{1}(r)\left[\partial_{i} v^{i}-b^{-1}\left(\partial_{t} b+v^{i} \partial_{i} b\right)\right]\left(-r^{2 z} f d t^{2}+2 b^{-\frac{\theta}{3(3-\theta)}} r^{z-1} d t d r\right) \\
& +2\left(F_{3}(r) \partial_{i} r_{0}+F_{5}(r) \partial_{i} b\right) d t\left(d x^{i}-v^{i} d t\right), \\
\varphi_{2}= & \sqrt{-\frac{2 \theta}{3(3-\theta)}} F_{1}(r)\left[\partial_{i} v^{i}-b^{-1}\left(\partial_{t} b+v^{i} \partial_{i} b\right)\right] .
\end{aligned}
$$

where $\theta$ must be negative integer for the metric before the dimensional reduction $d s_{D}^{2}$. The non-zero components of $v^{i}$ and $\mathcal{A}_{i}$ are introduced only for $i=1,2,3$ and the parameters $v^{i}, r_{0}, a, b$ and $\mathcal{A}_{\mu}$ are replaced by functions which depends on $x^{\mu}$ with $\mu=0, \cdots, 3$. We have redefined the coordinates $x^{\mu}$ as (3.10) in the previous section and hence the Hawking temperature is also rescaled as

$$
T=\frac{z+d-1-\theta}{4 \pi} e^{-\chi_{0}} r_{0}^{z}=\frac{z+d-1-\theta}{4 \pi} b^{\frac{\theta}{(d-1)(d-1-\theta)}} r_{0}^{z} .
$$

where we have written this relation in arbitrary dimension. The function $F(r)$ is given by

$$
\begin{aligned}
& F_{1}(r)=b^{-\frac{\theta}{3(3-\theta)}} \int d r \frac{r^{3-\theta}-r_{0}^{3-\theta}}{r\left(r^{z+3-\theta}-r_{0}^{z+3-\theta}\right)}, \\
& F_{2}(r)=\left(2(z-1) r^{z+3-\theta}-(z-5+\theta) r_{0}^{z+3-\theta}\right) \int d r \widehat{F}_{1}(r) \\
& F_{3}(r)=-2(z-1) a^{-1} b^{-\frac{\theta}{3(3-\theta)}} \int \frac{d r}{r^{6-z+\theta}} F_{2}(r) \\
& F_{4}(r)=\left(2(z-1) r^{z+3-\theta}-(z-5+\theta) r_{0}^{z+3-\theta}\right) \int d r \widehat{F}_{2}(r) \\
& F_{5}(r)=\int \frac{d r}{r^{6-z+\theta}}\left(-2(z-1) a^{-1} b^{-\frac{\theta}{3(3-\theta)}} F_{4}(r)+\frac{\theta}{3(3-\theta)} b^{-1-\frac{\theta}{3(3-\theta)}} r^{3-\theta}\right) \\
& \widehat{F}_{i}(r)=\frac{r^{7-2 \theta} \widetilde{F}_{i}(r)}{\left(r^{z+3-\theta}-r_{0}^{z+3-\theta}\right)\left[2(z-1) r^{z+3-\theta}-(z-5+\theta) r_{0}^{z+3-\theta}\right]^{2}} \\
& \widetilde{F}_{1}(r)=\frac{z+3-\theta}{2(z-1)} a_{0}^{r^{z-\theta}}\left(2(z-1)(5-\theta) r^{z+3-\theta} r_{0}^{2}-z(z+3-\theta) r^{5-\theta} r_{0}^{z}\right. \\
& \widetilde{F}_{2}(r)=-\frac{\theta}{6(3-\theta)(z-1)} a b^{-1} r^{-\theta-5} r_{0}^{-2 \theta}\left(-4(z-1)^{2} r_{0}^{2 \theta} r^{2 z+6}-r^{2 \theta} r_{0}^{2 z+6}(z-5+\theta)^{2}\right. \\
& \left.\quad+r^{\theta+5}(z+3-\theta)^{2} r_{0}^{\theta+2 z+1}+4(z-1)(z-5+\theta) r_{0}^{\theta+z+3} r^{\theta+z+3}\right)
\end{aligned}
$$


The first order solution of the gauge field is

$$
\begin{aligned}
\frac{1}{\sqrt{\mu}} A= & a(x)\left[b^{\frac{\theta}{3(3-\theta)}}\left(r^{z+3-\theta}-r_{0}^{z+3-\theta}(x)\right)-\frac{1}{3-\theta} r^{3-\theta} \partial_{i} v^{i}(x)\right] d t \\
& -a(x) r^{2-\theta} d r+\mathcal{A}_{\mu}(x) d x^{\mu}+\left(F_{2}(r) \partial_{i} r_{0}+F_{4}(r) \partial_{i} b\right)\left(d x^{i}-v^{i} d t\right),
\end{aligned}
$$

and $\phi_{1}$ has no correction term, and $\phi_{2}$ receives the correction term $\varphi_{2}$;

$$
\phi_{2}=-\frac{\theta}{(d-1) \tilde{\nu}} \log r+\frac{2}{\tilde{\nu}} \chi_{0}+\varphi_{2} .
$$

The solution above must satisfy the following constraints;

$$
\begin{aligned}
& 0=\partial_{t} a+v^{i} \partial_{i} a-a \partial_{i} v^{i}, \\
& 0=\partial_{t} r_{0}+v^{i} \partial_{i} r_{0}+\frac{1}{3-\theta} r_{0} \partial_{i} v^{i}, \\
& 0=\mathcal{F}_{t i}+v^{j} \mathcal{F}_{j i}+\frac{z+3-\theta}{2(z-1)} a b^{\frac{\theta}{3(3-\theta)}} r_{0}^{z+3-\theta}\left(z \frac{\partial_{i} r_{0}}{r_{0}}+\frac{\theta}{3(3-\theta)} \frac{\partial_{i} b}{b}\right)
\end{aligned}
$$

The hydrodynamic solution from the dimensional reduction also reproduce the result in the previous sections, if $b=a$.

\subsection{Higher-dimensional thermodynamics and hydrodynamics}

We first consider thermodynamics before the dimensional reduction. For $D$-dimensional space-time with arbitrary $D=d-\theta$, the energy, entropy and charge are given by

$$
\begin{aligned}
E_{D} & =\mathcal{E}_{D} V_{D-1}=\frac{D-1}{16 \pi G} r_{0}^{z+D-1} V_{D-1} \\
S_{D} & =s V_{D-1}=\frac{1}{4 G} r_{0}^{D-1} V_{D-1}, \\
N_{D} & =n V_{D-1}=\frac{z-1}{16 \pi G a} V_{D-1},
\end{aligned}
$$

where $V_{D-1}$ is the volume of the $(D-1)$-dimensional space. and 1st law of thermodynamics is expressed as

$$
d E_{D}=T_{D} d S_{D}-P_{D} d V_{D-1}+\mu_{D} d N_{D}
$$

and the temperature, pressure and chemical potential are calculated as

$$
\begin{aligned}
& T_{D}=\left(\frac{\partial E_{D}}{\partial S_{D}}\right)_{V_{D-1}, N_{D}}=\frac{D-1}{4 \pi} r_{0}^{z}, \\
& P_{D}=-\left(\frac{\partial E_{D}}{\partial V_{D-1}}\right)_{S_{D}, N_{D}}=\frac{z}{16 \pi G} r_{0}^{z+D-1}, \\
& \mu_{D}=\left(\frac{\partial E_{D}}{\partial N_{D}}\right)_{S_{D}, V_{D-1}}=0 .
\end{aligned}
$$

Here, temperature $T_{D}$ agrees with that for the local observer

$$
T_{D}=\frac{T}{\sqrt{g_{t t}}},
$$

where $T$ is the Hawking temperature of the black hole. 
Next, we consider the fluid in $D$-dimensional spacetime with $D=3-\theta$. The vielbein behaves near the boundary $r \rightarrow \infty$ as

$$
E_{(D)}^{0}=r^{z} \tau_{D}, \quad E_{(D)}^{i}=r \hat{e}_{D}^{i}
$$

where

$$
\begin{aligned}
& \tau_{D}=e^{-\chi_{0}} d t, \\
& \hat{e}_{D}^{i}=e^{-\chi_{0}}\left(d x^{i}-v^{i} d t\right), \quad(i=1,2,3) \\
& \hat{e}_{D}^{i}=e^{-\frac{6}{\theta} \chi_{0}} d x^{i} . \quad(i=4, \cdots, 3-\theta)
\end{aligned}
$$

This implies that the Newton-Cartan data on the boundary is given by

$$
\begin{aligned}
\tau_{D} & =e^{-\chi_{0}} d t \\
\hat{v}_{D}^{\mu} & =e^{\chi_{0}}\left(1, v^{i}, 0\right) \\
h_{D}^{\mu \nu} & =\operatorname{diag}\left(0, e^{2 \chi_{0}}, \cdots, e^{2 \chi_{0}}, e^{\frac{6}{\theta} \chi_{0}}, \cdots, e^{\frac{6}{\theta} \chi_{0}}\right),
\end{aligned}
$$

The stress-energy tensor on the boundary is calculated from the first order solution for metric before the dimensional reduction (6.20), in a similar fashion to section 4 as

$$
\begin{aligned}
\widetilde{T}_{D}{ }^{0}{ }_{0}= & \frac{1}{8 \pi G}\left(-\frac{3-\theta}{2} r_{0}^{z+3-\theta}-\frac{z-1}{a} b^{-\frac{\theta}{3(3-\theta)}} v^{i} \mathcal{A}_{i}\right) \\
\widetilde{T}_{D}{ }^{i}{ }_{0}= & \frac{1}{8 \pi G}\left[-\frac{z+3-\theta}{2} r_{0}^{z+3-\theta} v^{i}+\frac{z-1}{a} b^{-\frac{\theta}{3(3-\theta)}} v^{i} \mathcal{A}_{t}+\frac{1}{2} r_{0}^{3-\theta} \sigma_{D}{ }^{i}{ }_{j} v^{j}\right. \\
& \left.\quad+\frac{z(z+3-\theta)}{4(z-1)} b^{-\frac{\theta}{3(3-\theta)}} r_{0}^{2 z-\theta}\left(\partial_{i} r_{0}-\frac{\theta}{3(3-\theta) z} \frac{r_{0}}{b} \partial_{i} b\right)\right] \\
\widetilde{T}_{D}{ }^{0}{ }_{i}= & \frac{1}{8 \pi G} \frac{z-1}{a} b^{-\frac{\theta}{3(3-\theta)}} \mathcal{A}_{i}, \\
\widetilde{T}_{D}{ }^{i}{ }_{j}= & \frac{1}{8 \pi G}\left\{\frac{z}{2} r_{0}^{z+3-\theta} \delta_{i j}-\frac{1}{2} r_{0}^{3-\theta} \sigma_{D{ }^{i}{ }_{j}}+\frac{z-1}{a} b^{-\frac{\theta}{3(3-\theta)}}\left[v^{i} \mathcal{A}_{j}-\delta_{i j}\left(\mathcal{A}_{t}+v^{k} \mathcal{A}_{k}\right)\right]\right\}
\end{aligned}
$$

where the shear tensor is given by

$$
{\sigma_{D}}^{i}{ }_{j}=b^{-\frac{\theta}{3(3-\theta)}}\left(\partial_{i} v^{j}+\partial_{j} v^{i}-\frac{2}{3-\theta} \delta_{i j} \partial_{k} v^{k}\right)+\frac{2 \theta}{3(3-\theta)} b^{-\frac{\theta}{3(3-\theta)}-1}\left(\partial_{t} b+v^{k} \partial_{k} b\right) \delta_{i j},
$$

for $i, j=1, \cdots, d-1$.

This stress-energy tensor can be expressed in context of the Newton-Cartan theory as

$$
\begin{aligned}
\widetilde{T}_{D}{ }^{\mu}{ }_{\nu}= & \mathcal{E}_{D} \hat{v}_{D}^{\mu} \tau_{D \nu}+P_{D} \hat{h}_{D}{ }^{\mu}{ }_{\nu}-\kappa_{D} \tau_{D \nu} h_{D}^{\mu \rho}\left(\partial_{\rho}-\mathcal{G}_{\rho}\right) T_{D} \\
& -\eta_{D} \sigma_{D \rho \sigma} h_{D}^{\rho \mu} \hat{h}_{D}{ }^{\sigma}{ }_{\nu}+n \hat{v}_{D}^{\mu} \mathcal{A}_{\nu}-n \hat{v}_{D}{ }_{D} \mathcal{A}_{\rho} \delta^{\mu}{ }_{\nu}
\end{aligned}
$$

where we have defined

$$
\mathcal{G}_{\mu}=\left(\partial_{\nu} \tau_{D \mu}-\partial_{\mu} \tau_{D \nu}\right) \hat{v}_{D}^{\nu}
$$


The shear tensor can be expressed in terms of the Newton-Cartan geometry as

$$
\begin{aligned}
\sigma_{D \mu \nu} & =\hat{h}_{D \rho \nu} \widehat{D}_{\mu} \hat{v}_{D}^{\rho}+\hat{h}_{D \rho \mu} \widehat{D}_{\nu} \hat{v}_{D}^{\rho}-\frac{2}{3-\theta} \hat{h}_{D \mu \nu} \widehat{D}_{\rho} \hat{v}_{D}^{\rho} \\
& =£_{\hat{v}_{D}} \hat{h}_{D \mu \nu}-\frac{2}{3-\theta} \hat{h}_{D \mu \nu} \widehat{D}_{\rho} \hat{v}_{D}^{\rho},
\end{aligned}
$$

where $\widehat{D}_{\mu}$ is the covariant derivative in the Newton-Cartan geometry.

The energy density, pressure, particle number density and the temperature are given by

$$
\mathcal{E}_{D}=\frac{3-\theta}{16 \pi G} r_{0}^{z+3-\theta}, \quad P_{D}=\frac{z}{16 \pi G} r_{0}^{z+3-\theta}, \quad n=\frac{z-1}{16 \pi G} a^{-1}, \quad T_{D}=\frac{z+3-\theta}{4 \pi} r_{0}^{z} .
$$

The transport coefficients are read off as

$$
\eta_{D}=\frac{1}{16 \pi G} r_{0}^{3-\theta}, \quad \zeta_{D}=0, \quad \kappa_{D}=\frac{1}{8(z-1) G} r_{0}^{z+1-\theta} .
$$

The stress-energy tensor can also be expressed in terms of the energy current, momentum density, stress tensor and particle number current as

$$
\widetilde{T}_{D}{ }^{\mu}{ }_{\nu}=-\widetilde{\mathcal{E}}_{D}^{\mu} \tau_{D \nu}+\hat{v}_{D}^{\mu} \widetilde{\mathcal{P}}_{D \nu}+\widetilde{\mathcal{T}}_{D}{ }^{\mu}{ }_{\nu}+J_{D}^{\mu} \mathcal{A}_{\nu}-J_{D}^{\rho} \mathcal{A}_{\rho} \delta^{\mu}{ }_{\nu}
$$

where

$$
\begin{aligned}
\widetilde{\mathcal{E}}_{D}^{\mu} & =\mathcal{E}_{D} \hat{v}_{D}^{\mu}-\kappa_{D} h_{D}^{\mu \rho}\left(\partial_{\rho}-\mathcal{G}_{\rho}\right) T_{D}, \\
\widetilde{\mathcal{P}}_{\nu} & =0 \\
\widetilde{\mathcal{T}}_{D}{ }^{\mu}{ }_{\nu} & =P_{D} \hat{h}_{D}{ }^{\mu}{ }_{\nu}-\eta_{D} \sigma_{D \rho \sigma} h_{D}{ }^{\rho \mu} \hat{h}_{D}{ }_{\nu}{ }_{\nu}, \\
J_{D}^{\mu} & =n \hat{v}_{D}^{\mu} .
\end{aligned}
$$

The conservation equations in the Newton-Cartan theory is given by

$$
\begin{aligned}
\left(\widehat{D}_{\mu}-\mathcal{G}_{\mu}\right) \widetilde{\mathcal{E}}_{D}^{\mu} & =\hat{v}_{D}^{\mu}\left(F_{\mu \nu}^{\tau} \widetilde{\mathcal{E}}_{D}^{\nu}-\mathcal{F}_{\mu \nu} J_{D}^{\nu}\right)-\left(\widehat{D}_{\mu} \hat{v}_{D}{ }^{\nu}\right) \widetilde{\mathcal{T}}_{D}{ }^{\mu}{ }_{\nu}, \\
h_{D}^{\rho \mu}\left(\widehat{D}_{\nu}-\mathcal{G}_{\nu}\right) \widetilde{\mathcal{T}}_{D}{ }^{\nu}{ }_{\mu} & =h_{D}^{\rho \mu}\left[\hat{v}_{D}^{\nu} \widehat{D}_{\mu} \widetilde{\mathcal{P}}_{D \nu}-\widehat{D}_{\nu}\left(\hat{v}_{D}^{\nu} \widetilde{\mathcal{P}}_{D \mu}\right)+\mathcal{F}_{\mu \nu} J_{D}^{\nu}-F_{\mu \nu}^{\tau} \widetilde{\mathcal{E}}_{D}^{\nu}\right], \\
0 & =\left(\widehat{D}_{\mu}-\mathcal{G}_{\mu}\right) J_{D}^{\mu},
\end{aligned}
$$

where

$$
F_{\mu \nu}^{\tau}=\partial_{\mu} \tau_{D \nu}-\partial_{\nu} \tau_{D \mu}
$$

Then, they can be expressed in terms of the fluid variables as

$$
\begin{aligned}
& 0=\hat{v}_{D}^{\mu} \partial_{\mu} \mathcal{E}_{D}+\left(\mathcal{E}_{D}+P_{D}\right) \widehat{D}_{\mu} \hat{v}_{D}^{\mu}-\frac{1}{2} \eta_{D} \sigma_{D}{ }^{\mu}{ }_{\nu} \sigma_{D}{ }^{\nu}{ }_{\mu}-\left(\widehat{D}_{\mu}-2 \mathcal{G}_{\mu}\right)\left[\kappa_{D} h_{D}^{\mu \rho}\left(\partial_{\rho}-\mathcal{G}_{\rho}\right) T_{D}\right] \\
& 0=h_{D}^{\rho \nu} \partial_{\nu} P_{D}-h_{D}^{\rho \nu} \mathcal{G}_{\nu}\left(\mathcal{E}_{D}+P_{D}\right)-h_{D}^{\rho \mu} \mathcal{F}_{\mu \nu} J_{D}^{\nu}-h_{D}^{\mu \rho} h_{D}^{\nu \sigma}\left(\widehat{D}_{\sigma}-\mathcal{G}_{\sigma}\right)\left(\eta_{D} \sigma_{D \mu \nu}\right), \\
& 0=\widehat{D}_{\mu}\left(n \hat{v}_{D}^{\mu}\right) .
\end{aligned}
$$

It is straightforward to see that these equations are equivalent to the constraint equations in the bulk equations of motion. 


\subsection{Thermodynamics after the dimensional reduction}

For general $b$, we consider in the following the first law, where the thermodynamic variables are the entropy $S$, the $(d-1)$-dimensional volume ${ }^{13} V$ and the charge $N$, which is related variable $a$. We have already found that (in general $d$ )

$$
\begin{aligned}
E & =\mathcal{E} V=\frac{d-1-\theta}{16 \pi G} b^{\frac{\theta}{(d-1)(d-1-\theta)}} r_{0}^{z+d-1-\theta} V \\
S & =s V=\frac{1}{4 G} r_{0}^{d-1-\theta} V \\
N & =n V=\frac{z-1}{16 \pi G a} V .
\end{aligned}
$$

In general, $b$ is not related to the particle number density $n$, and hence the energy does not depend on the charge. Then the first law can be written as,

$$
d E=T d S-P d V+\mu d N
$$

where

$$
\begin{aligned}
T & =\left(\frac{\partial E}{\partial S}\right)_{V, N}=\frac{z+d-1-\theta}{4 \pi} b^{\frac{\theta}{(d-1)(d-1-\theta)}} r_{0}^{z}, \\
P & =-\left(\frac{\partial E}{\partial V}\right)_{S, N}=\frac{z}{16 \pi G} b^{\frac{\theta}{(d-1)(d-1-\theta)}} r_{0}^{z+d-1-\theta}, \\
\mu & =\left(\frac{\partial E}{\partial N}\right)_{S, V}=0 .
\end{aligned}
$$

The black hole solution also has another variable $b$, which is related to the scalar source as

$$
\tilde{\phi}_{2}=\tilde{\nu} \log b
$$

Then, by taking into this scalar source as additional thermodynamic variable, first law of thermodynamics may be expressed as

$$
d E=T d S-P d V+\mu d N+\left\langle\widetilde{\mathcal{O}}_{2}\right\rangle d \tilde{\phi}_{2},
$$

where $\left\langle\widetilde{\mathcal{O}}_{2}\right\rangle$ is vev of the dual operator to the scalar $\phi_{2}$, which is expressed as

$$
\left\langle\widetilde{\mathcal{O}}_{2}\right\rangle=\frac{\tilde{\nu}}{2} b^{\frac{\theta}{(d-1)(d-1-\theta)}}\left(\sum_{\mu=0}^{d-1} T_{D}{ }^{\mu}{ }_{\mu}+\frac{d-1}{\theta} \sum_{i=d}^{d-1-\theta} T_{D}{ }^{i}{ }_{i}\right)=-\frac{\tilde{\nu}}{2} \mathcal{E}-\tilde{\nu} \eta \bar{\vartheta}
$$

We will discuss this operator later.

In the previous section, we obtained the thermodynamics in the lower dimension, with one less variable. To recover it from the one here we must take a codimension one section

\footnotetext{
${ }^{13}$ Measured in the $d$-dimensional metric in the Einstein frame.
} 
of the thermodynamic variables. As argued already, the correct section involves taking $b=a$ above. With this constraint, we obtain

$$
\begin{aligned}
E & =\mathcal{E} V=\frac{d-1-\theta}{16 \pi G} a^{\frac{\theta}{(d-1)(d-1-\theta)}} r_{0}^{z+d-1-\theta} V \\
S & =s V=\frac{1}{4 G} r_{0}^{d-1-\theta} V \\
N & =n V=\frac{z-1}{16 \pi G a} V,
\end{aligned}
$$

and the first law takes the form

$$
d E=T d S-\widetilde{P} d V+\tilde{\mu} d N .
$$

The temperature, pressure and chemical potential are now given by

$$
\begin{aligned}
& T=\left(\frac{\partial E}{\partial S}\right)_{V, N}=\frac{z+d-1-\theta}{4 \pi} a^{\frac{\theta}{(d-1)(d-1-\theta)}} r_{0}^{z}, \\
& \widetilde{P}=-\left(\frac{\partial E}{\partial V}\right)_{S, N}=\frac{1}{16 \pi G}\left(z-\frac{\theta}{d-1}\right) a^{\frac{\theta}{(d-1)(d-1-\theta)}} r_{0}^{z+d-1-\theta}, \\
& \tilde{\mu}=\left(\frac{\partial E}{\partial N}\right)_{S, V}=-\frac{\theta}{(d-1)(z-1)} a^{1+\frac{\theta}{(d-1)(d-1-\theta)} r_{0}^{z+d-1-\theta} .}
\end{aligned}
$$

Note that this is exactly the thermodynamic quantities and the first law we obtained in the previous section.

The ward identity for the scaling symmetry is simply expressed as

$$
\left(z-\frac{\theta}{d-1}\right) \mathcal{E}=(d-1-\theta) P .
$$

\subsection{Hydrodynamics after the dimensional reduction}

The stress-energy tensor and the fluid equations in lower dimensional theory can be calculated straightforwardly. The stress-energy tensor is obtained as

$$
\begin{aligned}
& \widetilde{T}_{0}^{0}=\frac{1}{8 \pi G}\left(-\frac{3-\theta}{2} b^{\frac{\theta}{3(3-\theta)}} r_{0}^{z+3-\theta}-\frac{z-1}{a} v^{i} \mathcal{A}_{i}\right), \\
& \widetilde{T}^{i}{ }_{0}=\frac{1}{8 \pi G}\left[-\frac{z+3-\theta}{2} b^{\frac{\theta}{3(3-\theta)}} r_{0}^{z+3-\theta} v^{i}+\frac{z-1}{a} v^{i} \mathcal{A}_{t}+\frac{1}{2} r_{0}^{3-\theta} \sigma_{i j} v^{j}\right. \\
& -\frac{\theta}{3(3-\theta)} r_{0}^{3-\theta}\left[\partial_{j} v^{j}-b^{-1}\left(\partial_{t} b+v^{j} \partial_{j} b\right)\right] v^{i} \\
& \left.+\frac{z(z+3-\theta)}{4(z-1)} r_{0}^{2 z-\theta}\left(\partial_{i} r_{0}-\frac{\theta}{3(3-\theta) z} \frac{r_{0}}{b} \partial_{i} b\right)\right], \\
& \widetilde{T}^{0}{ }_{i}=\frac{1}{8 \pi G} \frac{z-1}{a} \mathcal{A}_{i} \\
& \widetilde{T}^{i}{ }_{j}=\frac{1}{8 \pi G}\left\{\frac{z}{2} b^{\frac{\theta}{3(3-\theta)}} r_{0}^{z+3-\theta} \delta_{i j}-\frac{1}{2} r_{0}^{3-\theta} \sigma_{i j}+\frac{z-1}{a} v^{i} \mathcal{A}_{j}-\delta_{i j}\left(\mathcal{A}_{t}+v^{k} \mathcal{A}_{k}\right)\right. \\
& \left.+\frac{\theta}{3(3-\theta)} r_{0}^{3-\theta}\left[\partial_{i} v^{i}-b^{-1}\left(\partial_{t} b+v^{i} \partial_{i} b\right)\right]\right\},
\end{aligned}
$$


and the expectation values of the dual operators of the dilatons $\phi_{1}$ and $\phi_{2}$ are calculated as

$$
\begin{gathered}
\left\langle\mathcal{O}_{1}\right\rangle=-\frac{\sqrt{(z-1)(3-\theta)}}{16 \pi G}\left[\frac{1}{2} b^{\frac{\theta}{3(3-\theta)}} r_{0}^{z+3-\theta}-\frac{2 \sqrt{2}}{a}\left(\mathcal{A}_{t}+v^{i} \mathcal{A}_{i}\right)\right] \\
\left\langle\mathcal{O}_{2}\right\rangle=-\frac{1}{16 \pi G}\left\{\sqrt{\frac{-\theta}{6(3-\theta)}\left[(z+2-\theta) b^{\frac{\theta}{3(3-\theta)}} r_{0}^{z+3-\theta}-\frac{2(z-1)}{a}\left(\mathcal{A}_{t}+v^{i} \mathcal{A}_{i}\right)\right]}\right. \\
\left.+\sqrt{-\frac{2 \theta}{3(3-\theta)}} r_{0}^{3-\theta}\left[\partial_{i} v^{i}-b^{-1}\left(\partial_{t} b+v^{i} \partial_{i} b\right)\right]\right\}
\end{gathered}
$$

For $b=a$, the results above agree with those in section 4 .

The stress-energy tensor after the dimensional reduction $\widetilde{T}_{\nu}^{\mu}$ can be expressed in terms of the fluid variables as

$$
\begin{aligned}
\widetilde{T}^{\mu}{ }_{\nu}= & \mathcal{E} \hat{v}^{\mu} \tau_{\nu}+P \hat{h}^{\mu}{ }_{\nu}-\kappa \tau_{\nu} h^{\mu \rho} \partial_{\rho} T-\eta \sigma_{a b} e_{a}^{\mu} \hat{e}_{\nu}^{b} \\
& -\zeta \bar{\vartheta} e_{a}^{\mu} \hat{e}_{\nu}^{a}+n \hat{v}^{\mu} \mathcal{A}_{\nu}-n \hat{v}^{\rho} \mathcal{A}_{\rho} \delta^{\mu}{ }_{\nu},
\end{aligned}
$$

where $\bar{\vartheta}$ is defined by

$$
\bar{\vartheta}=\partial_{i} v^{i}-b^{-1}\left(\partial_{t} b+v^{i} \partial_{i} b\right)
$$

which gives expansion for $b=1$. The energy density $\mathcal{E}$, pressure $P$, particle number density $n$ are same to those in section 6.2 ;

$$
\mathcal{E}=\frac{3-\theta}{16 \pi G} b^{\frac{\theta}{3(3-\theta)}} r_{0}^{z+3-\theta}, \quad P=\frac{z}{16 \pi G} b^{\frac{\theta}{3(3-\theta)}} r_{0}^{z+3-\theta}, \quad n=\frac{z-1}{16 \pi G} a^{-1} .
$$

The transport coefficients, heat conductivity $\kappa$ and shear viscosity $\eta$ are also read off as

$$
\kappa=\frac{1}{8(z-1) G} b^{-\frac{\theta}{3(3-\theta)}} r_{0}^{z+1-\theta}, \quad \quad \eta=\frac{1}{16 \pi G} r_{0}^{3-\theta} .
$$

For $b=a, \bar{\vartheta}$ vanishes but it does not mean that the fluid is incompressible, since $\bar{\vartheta}$ is not only the expansion but has extra terms. The expansion is non-zero but cancels with the extra terms, and hence, this implies that the bulk viscosity vanishes in the lower dimension. For the dimensional reduction with $b=1$ as in $(6.2), \bar{\vartheta}$ simply gives the expansion and hence the bulk viscosity $\zeta$ in that case is

$$
\zeta=-\frac{1}{8 \pi G} \frac{\theta}{3(3-\theta)} r_{0}^{3-\theta} .
$$

It should be noted that $\theta$ is negative for the dimensional reduction and hence $\zeta$ is positive.

The constraint equations can be written in terms of the fluid variables as

$$
\begin{aligned}
0= & \partial_{t} \mathcal{E}+v^{i} \partial_{i} \mathcal{E}+(\mathcal{E}+P) \partial_{i} v^{i} \\
& -\frac{1}{2} \eta \sigma_{i j} \sigma_{i j}-\zeta \bar{\vartheta} \partial_{i} v^{i}-\partial_{i}\left(\kappa \partial_{i} T\right)-\tilde{\nu}\left\langle\widetilde{\mathcal{O}}_{2}\right\rangle b^{-1}\left(\partial_{t} b+v^{i} \partial_{i} b\right), \\
0= & \partial_{i} P+J^{\mu} \mathcal{F}_{\mu i}-\partial_{j}\left(\eta \sigma_{i j}\right)-\partial_{i}(\zeta \bar{\vartheta})+\tilde{\nu}\left\langle\widetilde{\mathcal{O}}_{2}\right\rangle b^{-1} \partial_{i} b, \\
0= & \partial_{t} n+\partial_{j}\left(n v^{j}\right),
\end{aligned}
$$


where $\widetilde{\mathcal{O}}_{2}$ is the dual of the dilaton $\phi_{2}$ but without the contribution from the counter term $A^{2}$;

$$
\left\langle\widetilde{\mathcal{O}}_{2}\right\rangle=-\frac{1}{16 \pi G} \sqrt{\frac{-2 \theta}{3(3-\theta)}}\left\{\frac{3-\theta}{2} b^{\frac{\theta}{3(3-\theta)}} r_{0}^{z+3-\theta}+r_{0}^{3-\theta}\left[\partial_{i} v^{i}-b^{-1}\left(\partial_{t} b+v^{i} \partial_{i} b\right)\right]\right\} .
$$

This expression is consistent to the dimensional reduction from the higher dimensional fluid;

$$
\left\langle\widetilde{\mathcal{O}}_{2}\right\rangle=\frac{\tilde{\nu}}{2} b^{\frac{\theta}{3(3-\theta)}}\left(\sum_{\mu=0}^{3} T_{D}{ }^{\mu}{ }_{\mu}+\frac{3}{\theta} \sum_{i=4}^{3-\theta} T_{D}{ }^{i}{ }_{i}\right)=-\frac{\tilde{\nu}}{2} \mathcal{E}-\tilde{\nu} \eta \bar{\vartheta}
$$

where $T_{D}{ }_{\nu}{ }_{\nu}$ is the stress-energy tensor of the fluid before the dimensional reduction. Thus, $\left\langle\widetilde{\mathcal{O}}_{2}\right\rangle$ is related to the other fluid variables if the fluid is obtained by the dimensional reduction. It should be noted that the constant mode of $\phi_{2}$ is given by $\tilde{\nu} \log b$, and hence,the contribution from $\widetilde{\mathcal{O}}_{2}$ is interpreted as the coupling to the external source of $\phi_{2}$. Contrary to the single dilaton case in the previous sections, the variable $b$ is independent from the fluid variables and is interpreted as an external field. The transport coefficients also depend on temperature and external field $b$, but are independent of the particle number density.

The energy $\mathcal{E}$, stress tensor $\widehat{\mathcal{T}}^{i}{ }_{j}$ and scalar operator $\widetilde{\mathcal{O}}_{2}$ satisfy the following condition;

$$
0=-\left(z-\frac{\theta}{d-1}\right) \mathcal{E}+\left(1-\frac{\theta}{d-1}\right) \widehat{\mathcal{T}}^{i}{ }_{i}-(d-1-\theta) \tilde{\nu}\left\langle\widetilde{\mathcal{O}}_{2}\right\rangle
$$

where the trace of the Milne invariant stress tensor is given by

$$
\widehat{\mathcal{T}}_{i}{ }_{i}=(d-1)(P-\zeta \bar{\vartheta})
$$

The above condition is nothing but the Ward identity of the Lifshitz scaling symmetry with the hyperscaling-violation. The coefficients of $\mathcal{E}, P$ and $\tilde{\nu}\left\langle\widetilde{\mathcal{O}}_{2}\right\rangle$ equal to the scaling dimensions of $t, x^{i}$ and $b$ with appropriate signs, respectively.

If the fluid satisfies the condition $\bar{\vartheta}=0$, the fluid equations (6.104) and (6.105) can be rewritten as

$$
\begin{aligned}
& 0=\partial_{t} \mathcal{E}+v^{i} \partial_{i} \mathcal{E}+(\mathcal{E}+\widetilde{P}) \partial_{i} v^{i}-\frac{1}{2} \eta \sigma_{i j} \sigma_{i j}-\partial_{i}\left(\kappa \partial_{i} T\right) \\
& 0=\partial_{i} \widetilde{P}-\tilde{n} \partial_{i} \tilde{\mu}+J^{\mu} \mathcal{F}_{\mu i}-\partial_{j}\left(\eta \sigma_{i j}\right)
\end{aligned}
$$

where

$$
\begin{aligned}
& \widetilde{P}=P+\tilde{\mu} \tilde{n}=\frac{1}{16 \pi G}\left(z-\frac{\theta}{d-1}\right) b^{\frac{\theta}{(d-1)(d-1-\theta)}} r_{0}^{z+d-1-\theta}, \\
& \tilde{n}=\frac{(z-1)}{16 \pi G b}, \\
& \tilde{\mu}=-\tilde{n}^{-1} \tilde{\nu}\left\langle\widetilde{\mathcal{O}}_{2}\right\rangle=-\frac{\theta}{(d-1)(z-1)} b^{1+\frac{\theta}{(d-1)(d-1-\theta)}} r_{0}^{z+d-1-\theta} .
\end{aligned}
$$

The Ward identity of the Lifshitz scaling symmetry can also be expressed in terms of $\widetilde{P}$ as

$$
\left(z-\frac{\theta}{d-1}\right) \mathcal{E}=(d-1-\theta) \widetilde{P} .
$$


For $b=a$, these equations agree with those in the previous section. The above effective pressure (6.113) and chemical potential (6.115) agree with those in the thermodynamic relation (6.90) and (6.91), respectively. Then, the thermodynamic relations, fluid equations and Ward identity, as well as the stress-energy tensor reproduce the result in the previous section.

The result in this section is a generalization of [72] to non-relativistic and $z \neq 1$ cases. The contributions from the gauge field and $\phi_{1}$ vanish for $z=1$ limit in which the Lifshitz black hole geometry becomes the Schwarzschild-AdS. The hydrodynamic ansatz should be given by using the lorentz boost for $z=1$, and hence the fluid will be relativistic. The results in this section are not well defined in $z=1$ limit, since the ansatz is given by using the the Galilean boost. The non-relativistic fluid which is obtained in this section agrees with non-relativistic limit of [72] for $z=1$.

\section{Lifshitz hydrodynamics on conformally flat background}

We have introduced the redefinition of the boundary coordinate before replacing the parameters by slowly varying functions. This coordinate redefinition is introduced to take the flat space background on the boundary. Here, we show that naive hydrodynamic ansatz without such a coordinate redefinition gives fluids on the non-trivial but conformally flat background.

It can be calculated straightforwardly in a similar fashion to section 3 but without introducing the coordinate redefinition (3.10). Then, the first order constraint equations, which are equivalent to the fluid equations in the perfect fluid limit, are obtained as

$$
\begin{aligned}
& 0=\partial_{t} a+v^{i} \partial_{i} a-a\left(1-\frac{\theta}{3}\right) \partial_{i} v^{i}, \\
& 0=\partial_{t} r_{0}+v^{i} \partial_{i} r_{0}+\frac{1}{3} r_{0} \partial_{i} v^{i}, \\
& 0=\mathcal{F}_{t i}+v^{j} \mathcal{F}_{j i}+\frac{z(z+3-\theta)}{2(z-1)} a r_{0}^{z+2-\theta} \partial_{i} r_{0} .
\end{aligned}
$$

These equations are different from (3.36)-(3.38), and as we will explain below, the difference can be interpreted as the effect of the non-trivial background geometry at the boundary. It is natural to expect that fluid variables as energy density, pressure and particle number density does not affected by the background geometry. In fact, we can calculate the stressenergy tensor straightforwardly and they are read off as

$$
\begin{aligned}
\mathcal{E} & =\frac{3-\theta}{16 \pi G} a^{\frac{\theta}{3(3-\theta)}} r_{0}^{z+3-\theta}, & P & =\frac{1}{16 \pi G}\left(z-\frac{\theta}{d-1}\right) a^{\frac{\theta}{3(3-\theta)}} r_{0}^{z+3-\theta}, \\
n & =\frac{z-1}{8 \pi G} a^{-1}, & \mu & =-\frac{\theta}{3(z-1)} a^{1+\frac{\theta}{3(3-\theta)}} r_{0}^{z+3-\theta} .
\end{aligned}
$$

which are same to (5.8).

In order to consider the fluid mechanics in the non-trivial background, we first introduce the fluid velocity field $u^{\mu}$ which is normalized as

$$
1=\tau_{\mu} u^{\mu}
$$


where the timelike vielbein is given by

$$
\begin{aligned}
\tau & =e^{\chi_{0}} d t, \\
e^{\chi_{0}} & =a^{-\frac{\theta}{3(3-\theta)}}
\end{aligned}
$$

and hence the normalized velocity field $u^{\mu}$ is

$$
u^{t}=e^{-\chi_{0}}, \quad u^{i}=e^{-\chi_{0}} v^{i} .
$$

The constraint equations (7.1)-(7.3) can be expressed as

$$
\begin{aligned}
& 0=u^{\mu} \partial_{\mu} \mathcal{E}+(\mathcal{E}+P) D_{\mu} u^{\mu}, \\
& 0=\partial_{i} P-n \partial_{i} \mu+(\mathcal{E}+P) \partial_{i} \chi_{0}+J^{\mu} \mathcal{F}_{\mu i}, \\
& 0=D_{\mu} J^{\mu}
\end{aligned}
$$

where the particle number current $J^{\mu}$ is defined by

$$
J^{\mu}=n u^{\mu} .
$$

Eq. (7.9)-(7.11) are nothing but the fluid equations in the perfect fluid limit in NewtonCartan theory, and generalization to the first order fluid is straightforward.

The first order stress-energy tensor is obtained in the following form;

$$
\widehat{T}^{\mu}{ }_{\nu}=\mathcal{E} u^{\mu} \tau_{\nu}+(P-n \mu) \hat{h}^{\mu}{ }_{\nu}-\tilde{\kappa} \tau_{\nu} h^{\mu \rho} \partial_{\rho} T-\tilde{\eta} \sigma_{a b} e_{a}^{\mu} \hat{e}_{\nu}^{b}+n u^{\mu} \mathcal{A}_{\nu}-n u^{\rho} \mathcal{A}_{\rho} \delta^{\mu}{ }_{\nu},
$$

where the transport coefficients are given by

$$
\tilde{\kappa}=\frac{1}{8(z-1) G} r_{0}^{z+1-\theta}, \quad \tilde{\eta}=\frac{1}{16 \pi G} a^{\frac{\theta}{3(3-\theta)}} r_{0}^{3-\theta}, \quad \zeta=0 .
$$

The bulk viscosity is zero as for the flat background (5.13). The difference of heat conductivity would come from the difference of the temperature. In this case, the Hawking temperature is simply given by (2.22) on the contrary to that in section 3 where the temperature is rescaled due to the coordinate transformation. In the curved background, this should be expressed in terms of the local temperature $T_{T}=e^{-\chi_{0}} T$ as

$$
\tilde{\kappa} \partial_{\mu} T=\kappa\left(\partial_{\mu}-\mathcal{G}_{\mu}\right) T_{T},
$$

and then, the heat conductivity is same as (5.13);

$$
\kappa=\frac{1}{8(z-1) G} a^{-\frac{\theta}{3(3-\theta)}} r_{0}^{z+1-\theta},
$$

The difference of the shear viscosity implies that the shear tensor must be written in terms of the normalized velocity field $u^{\mu}$;

$$
\hat{\sigma}_{\mu \nu}=\hat{h}_{\rho \nu} D_{\mu} u^{\rho}+\hat{h}_{\rho \mu} D_{\nu} u^{\rho}-\frac{2}{3} \hat{h}_{\mu \nu} D_{\rho} u^{\rho} .
$$

Then, the shear can be written as

$$
\tilde{\eta} \sigma_{a b} e_{a}^{\mu} \hat{e}_{\nu}^{b}=\eta \hat{\sigma}_{\rho \sigma} h^{\mu \rho} h_{\nu}^{\sigma}
$$

Then, the shear viscosity $\eta$ equals to (5.13);

$$
\eta=\frac{1}{16 \pi G} r_{0}^{3-\theta} .
$$




\subsection{Dimensional reduction for conformally flat background}

We can also consider the naive hydrodynamic ansatz for the dimensional reduction from the higher dimensional Lifshitz geometry. In this case, we can see the effects of the conformal factor in the metric more explicitly. The perfect fluid limit of the fluid equations are obtained as

$$
\begin{aligned}
& 0=a^{-1}\left(\partial_{t} a+v^{i} \partial_{i} a\right)+\frac{\theta}{3-\theta} b^{-1}\left(\partial_{t} b+v^{i} \partial_{i} b\right)-\partial_{i} v^{i}, \\
& 0=r_{0}^{-1}\left(\partial_{t} r_{0}+v^{i} \partial_{i} r_{0}\right)+\frac{\theta}{(3-\theta)^{2}} b^{-1}\left(\partial_{t} b+v^{i} \partial_{i} b\right)+\frac{1}{3-\theta} \partial_{i} v^{i}, \\
& 0=\mathcal{F}_{t i}+v^{j} \mathcal{F}_{j i}+\frac{z(z+3-\theta)}{2(z-1)} a r_{0}^{z+2-\theta} \partial_{i} r_{0} .
\end{aligned}
$$

where $b$ comes from the effects of the non-trivial background;

$$
e^{\chi_{0}}=b^{-\frac{\theta}{3(3-\theta)}} .
$$

The fluid variables are same to $(6.101)$

$$
\mathcal{E}=\frac{3-\theta}{16 \pi G} b^{\frac{\theta}{3(3-\theta)}} r_{0}^{z+3-\theta}, \quad P=\frac{z}{16 \pi G} b^{\frac{\theta}{3(3-\theta)}} r_{0}^{z+3-\theta}, \quad n=\frac{z-1}{8 \pi G} a^{-1},
$$

and the fluid equations can be written in terms of the normalized velocity field $u^{\mu}$ but now $\chi_{0}$ is independent from the particle number density $n \sim 1 / a$;

$$
\begin{aligned}
& 0=u^{\mu} \partial_{\mu} \mathcal{E}+(\mathcal{E}+P) D_{\mu} u^{\mu}+\tilde{\nu} \widetilde{\mathcal{O}}_{2} b^{-1} u^{\mu} \partial_{\mu} b, \\
& 0=\partial_{i} P+(\mathcal{E}+P) \partial_{i} \chi_{0}+J^{\mu} \mathcal{F}_{\mu i}-\tilde{\nu} \widetilde{\mathcal{O}}_{2} b^{-1} \partial_{i} b, \\
& 0=D_{\mu} J^{\mu}
\end{aligned}
$$

The first order stress-energy tensor is given by

$$
\begin{aligned}
\widehat{T}^{\mu}{ }_{\nu}= & \mathcal{E} u^{\mu} \tau_{\nu}+P \hat{h}^{\mu}{ }_{\nu}-\tilde{\kappa} \tau_{\nu} h^{\mu \rho} \partial_{\rho} T-\eta \hat{\sigma}_{\rho \sigma} h^{\mu \rho} h^{\sigma}{ }_{\nu} \\
& -\zeta\left[\partial_{i} v^{i}-\frac{3}{3-\theta} b^{-1}\left(\partial_{t} b+v^{i} \partial_{i} b\right)\right] e_{a}^{\mu} \hat{e}_{\nu}^{a}+n u^{\mu} \mathcal{A}_{\nu}-n u^{\rho} \mathcal{A}_{\rho} \delta^{\mu}{ }_{\nu} .
\end{aligned}
$$

In this case, the expansion appears in the combination of

$$
\partial_{i} v^{i}-\frac{3}{3-\theta} b^{-1}\left(\partial_{t} b+v^{i} \partial_{i} b\right),
$$

and it vanishes for $b=a$ by substituting the constraint equation. For $b=1$, the background becomes flat and hence this agrees with (6.99).

\section{Acknowledgments}

We would like to thank J. deBoer, N. Obers for discussions. We would like to thank especially J. Hartong for enlightening discussions and for critical comments on the manuscript.

This work was supported in part by European Union's Seventh Framework Programme under grant agreements (FP7-REGPOT-2012-2013-1) no 316165 and the Advanced ERC grant SM-grav, No 669288. The work is also supported in part by the Ministry of Science and Technology, R.O.C. (project no. 104-2112-M-002 -003 -MY3) and by National Taiwan University (project no. 105R8700-2). 


\section{A Notations}

\section{Variables defined in the bulk (gravity) side.}

- $v^{i}$ : boost parameter introduced into the (static) black hole geometry in (3.11).

- $\mathcal{A}_{\mu}$ : the constant part of the gauge field, which is defined in (3.16). This corresponds to the Milne boost-invariant gauge field in the Newton-Cartan theory $\widehat{B}$, or equivalently, $B$ in the holographic frame $\bar{v}^{\mu}=\hat{v}^{\mu}$. In this paper, it is sometimes expressed as the 1 -form $\mathcal{A}=\mathcal{A}_{\mu} d x^{\mu}$.

- $\tau_{\mu}$ : the timelike vielbein on the boundary which is defined up to the factor of $e^{\chi} r^{z}$, and given by (4.3) in $r \rightarrow \infty$. This corresponds to the timelike unit normal which defines the time direction in the Newton-Cartan theory. It is automatically invariant under the Milne boost.

- $\hat{e}_{\mu}^{a}$ : the spacelike vielbein on the boundary which is defined up to the factor of $e^{\chi} r$, and given by (4.3) in $r \rightarrow \infty$. This corresponds to the spacelike vielbein in the Newton-Cartan theory if we take the holographic frame $\bar{v}^{\mu}=\hat{v}^{\mu}$.

- $\hat{v}^{\mu}$ : the timelike inverse vielbein on the boundary which is defined up to the factor $e^{-\chi} r^{-z}$, and given by (4.6) in $r \rightarrow \infty$. This corresponds to the inverse timelike vielbein in the Newton-Cartan theory if we take the holographic frame $\bar{v}^{\mu}=\hat{v}^{\mu}$, since the holographic frame is defined by $\bar{v}^{\mu}=\hat{v}^{\mu}$.

- $e_{a}^{\mu}$ : the spacelike inverse vielbein on the boundary which is defined up to the factor of $e^{-\chi} r^{-1}$, and given by (4.6) in $r \rightarrow \infty$. This corresponds to the spacelike inverse vielbein in the Newton-Cartan theory. It is automatically invariant under the Milne boost.

- $\widetilde{T}^{\mu}{ }_{\nu}$ : the energy-momentum tensor calculated from the black hole geometry (3.24), which is defined by $(4.13)$

- $\widetilde{\mathcal{E}}^{\mu}$ : the energy current which is calculated from the black hole geometry (3.24). It is defined by (5.18).

- $\widetilde{\mathcal{P}}_{\mu}$ : the momentum density which is calculated from the black hole geometry (3.24). It is defined by (5.18).

- $\widetilde{\mathcal{T}}^{\mu}{ }_{\nu}$ : the stress tensor which is calculated from the black hole geometry (3.24). It is defined by (5.18).

- $J^{\mu}$ : the particle number current which is calculated from the black hole geometry (3.24). It is given by (5.22). 
Variables defined in the (boundary) Newton-Cartan theory. The following variables in the Newton-Cartan theory are appears in section 5. See appendix B for more details.

- $\bar{v}^{\mu}$ : the timelike inverse vielbein in Newton-Cartan theory. The timelike inverse vielbein is not invariant under the Milne boost. In the literature it is sometimes called "velocity" but must be distinguished from the velocity of the fluid.

- $h^{\mu \nu}$ : the induced contravariant metric on the time-slice. It is invariant under the Milne boost.

- $\bar{h}_{\mu \nu}$ : the induced covariant metric on the time-slice. It is not invariant under the Milne boost.

- $\hat{h}_{\mu \nu}$ : the induced metric on the time-slice in the holographic frame $\bar{v}^{\mu}=\hat{v}^{\mu}$. It is given by $\hat{h}_{\mu \nu}=\hat{e}_{a}^{\mu} \hat{e}_{a}^{\nu}$. We also have $\hat{h}^{\mu}{ }_{\nu}=h^{\mu \rho} \hat{h}_{\rho \nu}$ and $\hat{h}^{\mu \nu}=h^{\mu \rho} \hat{h}_{\rho \sigma} h^{\sigma \nu}=h^{\mu \nu}$.

- $\bar{e}_{\mu}^{a}$ : the spacelike vielbein. It is given by $\bar{e}_{\mu}^{a}=\operatorname{diag}(0,1,1,1)$ for a 4 -dim space-time. It satisfies $\bar{h}_{\mu \nu}=\bar{e}_{\mu}^{a} \bar{e}_{\nu}^{a}$.

- $\bar{P}_{\nu}^{\mu}$ : the projection to the spatial direction, which is defined by $e_{a}^{\mu} \bar{e}_{\nu}^{a}$, and satisfies $\bar{P}_{\nu}^{\mu}=\bar{h}_{\nu}^{\mu}=h^{\mu \rho} \bar{h}_{\rho \nu}$

- $u^{\mu}$ : the fluid velocity field. It equals to the vielbein in the holographic frame $\hat{v}^{\mu}$.

- $B_{\mu}$ : the gauge field in the Newton-Cartan theory. It is not invariant under the Milne boost.

- $\mathcal{E}^{\mu}$ : the energy current in the Newton-Cartan theory. For fluids, it is given by (5.23).

- $\mathcal{P}_{\mu}$ : the momentum density in the Newton-Cartan theory. For fluids, it is given by (5.24).

- $\mathcal{T}^{\mu}{ }_{\nu}$ : the generic stress tensor in the Newton-Cartan theory. For fluids, it is given by (5.25).

- $\mathcal{J}^{\mu}$ : the mass current in the Newton-Cartan theory. For fluids, it is given by (5.26).

- $\bar{T}^{\mu}{ }_{\nu}$ : generic stress-energy tensor which is constructed from $\mathcal{E}^{\mu}, \mathcal{P}_{\mu}$ and $\mathcal{T}^{\mu}{ }_{\nu}$ in (5.28).

- $\widehat{B}$ : a Milne boost invariant combination for the gauge field, or equivalently, the gauge field in the holographic frame $\bar{v}^{\mu}=\hat{v}^{\mu}$. It is defined by (5.31).

- $\widehat{\mathcal{E}}^{\mu}$ : a Milne boost invariant combination for the energy current. or equivalently, the energy current in the holographic frame $\bar{v}^{\mu}=\hat{v}^{\mu}$. It is defined by (5.40).

- $\widehat{\mathcal{P}}_{\mu}$ : a Milne boost invariant combination for the stress tensor, or equivalently, the momentum density in the holographic frame $\bar{v}^{\mu}=\hat{v}^{\mu}$. It is defined by (5.40). 
- $\widehat{\mathcal{T}}^{\mu}{ }_{\nu}$ : a Milne boost invariant combination for the stress tensor, or equivalently, the stress tensor in the holographic frame $\bar{v}^{\mu}=\hat{v}^{\mu}$. It is defined by (5.40).

- $T^{\mu}{ }_{\nu}$ : a Milne boost invariant combination of the stress-energy tensor, or equivalently the stress-energy tensor in the holographic frame $\bar{v}^{\mu}=\hat{v}^{\mu}$. It is defined by (5.33) and can be decomposed into $\widehat{\mathcal{E}}^{\mu}, \widehat{\mathcal{P}}_{\mu}$ and $\widehat{\mathcal{T}}^{\mu}{ }_{\nu}$.

\section{B The Newton-Cartan formalism}

Here, we briefly review on the Newton-Cartan formalism. The metric on the Galilei spacetime is defined by a 1-form $\tau_{\mu}$ and a contravariant symmetric tensor $h^{\mu \nu}$. The time direction of the Galilei spacetime is defined by the 1 -form $\tau_{\mu}$, and $h^{\mu \nu}$ is the spatial inverse metric on the timeslice. They satisfy the following orthogonality condition;

$$
\tau_{\mu} h^{\mu \nu}=0 \text {. }
$$

Next, we introduce the covariant derivative on the Galilei spacetime. We impose the condition that the Galilei date $\left(\tau_{\mu}, h^{\mu \nu}\right)$ are constant under the covariant derivative

$$
D_{\mu} \tau_{\nu}=0, \quad D_{\rho} h^{\mu \nu}=0 .
$$

On the contrary to Einstein gravity, the above condition does not determine the Galilei connection uniquely. We further introduce a contravariant vector $\bar{v}^{\mu}$, which satisfies the following normalization condition;

$$
\tau_{\mu} \bar{v}^{\mu}=1 .
$$

The vector $\bar{v}^{\mu}$ is sometimes referred to as velocity field but not to be confused with that of fluids. We also define the spatial covariant metric $\bar{h}_{\mu \nu}$ by using the following conditions with $\bar{v}^{\mu}$;

$$
\bar{h}_{\mu \nu} \bar{v}^{\mu}=0, \quad \bar{h}_{\mu \rho} h^{\rho \nu}=\bar{P}_{\mu}^{\nu}=\delta_{\mu}{ }^{\nu}-\tau_{\mu} \bar{v}^{\nu}
$$

We impose the spatial torsion free condition,

$$
\bar{h}_{\sigma \rho} T_{\mu \nu}^{\rho}=0
$$

where $T_{\mu \nu}^{\rho}=\Gamma_{\mu \nu}^{\rho}-\Gamma_{\nu \mu}^{\rho}$. Then the Newton-Cartan connection is determined up to a 2 -form $H_{\mu \nu}$ as

$$
\Gamma_{\mu \nu}^{\rho}=\bar{v}^{\rho} \partial_{\mu} \tau_{\nu}+\frac{1}{2} h^{\rho \sigma}\left(\partial_{\mu} \bar{h}_{\nu \sigma}+\partial_{\nu} \bar{h}_{\mu \sigma}-\partial_{\sigma} \bar{h}_{\mu \nu}\right)+\frac{1}{2} h^{\rho \sigma}\left(\tau_{\mu} H_{\nu \sigma}+\tau_{\nu} H_{\mu \sigma}\right) .
$$

In general, the Newton-Cartan connection has the torsion;

$$
T_{\mu \nu}^{\rho}=\bar{v}^{\rho}\left(\partial_{\mu} \tau_{\nu}-\partial_{\nu} \tau_{\mu}\right)
$$

Without the spatial torsion free condition, (B.5), the connection can be more general [74]. For example, we can define the Milne boost invariant connection [71, 74]

$$
\Gamma_{B \mu \nu}^{\rho}=v_{B}^{\rho} \partial_{\mu} \tau_{\nu}+\frac{1}{2} h^{\rho \sigma}\left(\partial_{\mu} h_{\nu \sigma}^{B}+\partial_{\nu} h_{\mu \sigma}^{B}-\partial_{\sigma} h_{\mu \nu}^{B}\right)
$$


where

$$
v_{B}^{\mu}=\bar{v}^{\mu}-h^{\mu \nu} B_{\nu}, \quad h_{\mu \nu}^{B}=\bar{h}_{\mu \nu}+\tau_{\mu} B_{\nu}+\tau_{\nu} B_{\mu},
$$

and $B$ is related to $H$ in (B.6) by $H=d B$, which we will discuss, soon. The connection (B.8) has non-zero spatial torsion;

$$
\bar{h}_{\sigma \rho} T_{B \mu \nu}^{\rho}=\bar{h}_{\sigma \rho} v_{B}^{\rho}\left(\partial_{\mu} \tau_{\nu}-\partial_{\nu} \tau_{\mu}\right)=-\bar{P}_{\sigma}^{\rho} B_{\rho}\left(\partial_{\mu} \tau_{\nu}-\partial_{\nu} \tau_{\mu}\right),
$$

or equivalently, (B.8) satisfies the modified spatial torsion free condition, $h_{\sigma \rho}^{B} T_{B \mu \nu}^{\rho}=0$ instead of (B.5). Here, the spatial torsion free connection (B.6) is sufficient for our purposes and hence we do not consider other connections as (B.8).

The curvature is defined by using the commutator of the covariant derivative and given by

$$
\mathcal{R}^{\mu}{ }_{\nu \rho \sigma}=\partial_{\rho} \Gamma_{\nu \sigma}^{\mu}-\partial_{\sigma} \Gamma_{\nu \rho}^{\mu}+\Gamma_{\alpha \rho}^{\mu} \Gamma_{\nu \sigma}^{\alpha}-\Gamma_{\alpha \sigma}^{\mu} \Gamma_{\nu \rho}^{\alpha} .
$$

For the torsion free case, $d \tau=0$, we impose the Newtonian condition

$$
\left.\mathcal{R}^{[\mu}{ }_{(\nu}^{\rho]}\right]_{\sigma)}=0,
$$

where $[\cdots]$ and $(\cdots)$ in the indices stand for the antisymmetric part and symmetric part, respectively. The above condition implies that the 2 -form $H_{\mu \nu}$ must be closed, $d H=0$. Then, $H$ is interpreted as the field strength of a gauge field; $H=d B$. For Torsional case, $d \tau \neq 0$, no appropriate generalization of the Newtonian condition is known. Here, we simply impose the condition $H=d B$ even for $d \tau \neq 0$, as is proposed in [71]. To summarize, the Newton-Cartan geometry is described by the Newton-Cartan data, $\tau_{\mu}, h^{\mu \nu}, \bar{v}^{\mu}$ and $B_{\mu}$.

In the Newton-Cartan data, $\bar{v}^{\mu}$ and $B_{\mu}$ are introduced to defined the Newton-Cartan connection. This means that $\bar{v}^{\mu}$ and $B_{\mu}$ themselves are transforming quantities but $\tau_{\mu}, h^{\mu \nu}$ and the Newton-Cartan connection do not. Two pairs $\left(\bar{v}^{\mu}, B_{\mu}\right)$ and $\left(\bar{v}^{\prime \mu}, B_{\mu}^{\prime}\right)$ are equivalent if they give the same Newton-Cartan connection. The transformation from $\left(\bar{v}^{\mu}, B_{\mu}\right)$ to $\left(\bar{v}^{\prime \mu}, B_{\mu}^{\prime}\right)$ gives an internal symmetry of the Newton-Cartan theory. The Newton-Cartan connection is invariant under the following transformation, [74];

$$
\begin{aligned}
\bar{v}^{\mu} \rightarrow \bar{v}^{\prime \mu} & =\bar{v}^{\mu}+h^{\mu \nu} V_{\nu}, \\
B \rightarrow B^{\prime} & =B+\bar{P}_{\mu}^{\nu} V_{\nu} d x^{\mu}-\frac{1}{2} h^{\mu \nu} V_{\mu} V_{\nu} \tau_{\rho} d x^{\rho} .
\end{aligned}
$$

This transformation is known as the Milne boost and $V_{\mu}$ is a vector which parametrize the Milne boost. It should be noted that the covariant spatial metric $\bar{h}_{\mu \nu}$ is defined in terms of $\bar{v}^{\mu}$ and is not invariant under the Milne boost. It transforms as

$$
\bar{h}_{\mu \nu}^{\prime}=\bar{h}_{\mu \nu}-\left(\tau_{\mu} \bar{P}_{\nu}^{\rho}+\tau_{\nu} \bar{P}_{\mu}^{\rho}\right) V_{\rho}+\tau_{\mu} \tau_{\nu} h^{\rho \sigma} V_{\rho} V_{\sigma} .
$$

Next, we consider the conservation law in the Newton-Cartan theory. The energy current $\mathcal{E}^{\mu}$, momentum density $\mathcal{P}_{\mu}$, stress tensor $\mathcal{T}_{\mu \nu}$ and mass current $\mathcal{J}^{\mu}$ are given by the variation of the action with respect to the Newton-Cartan data $\tau_{\mu}, h^{\mu \nu}, \bar{v}^{\mu}$ and $B_{\mu}$;

$$
\delta S=\int d^{d} x \sqrt{\gamma}\left[\delta \tau_{\mu} \mathcal{E}^{\mu}+\delta \bar{v}^{\mu} \mathcal{P}_{\mu}+\delta h^{\mu \nu} \mathcal{T}_{\mu \nu}+\delta B_{\mu} \mathcal{J}^{\mu}\right]
$$


where the variation $\delta \tau_{\mu}$ is arbitrary but $\delta \bar{v}^{\mu}$ and $\delta h^{\mu \nu}$ are only the variations which satisfy the orthogonality and normalization conditions, (B.1) and (B.3), namely, $\delta \bar{v}^{\mu}=P_{\nu}^{\mu} \delta \bar{v}^{\nu}$, etc. Invariance under the coordinate transformation gives the following conservation equations

$$
\begin{aligned}
\left(D_{\mu}-\mathcal{G}_{\mu}\right) \mathcal{E}^{\mu} & =\bar{v}^{\mu}\left(H_{\mu \nu} \mathcal{J}^{\nu}+K_{\mu \nu} \mathcal{E}^{\nu}\right)-\frac{1}{2}\left(h^{\mu \rho} D_{\rho} \bar{v}^{\nu}+h^{\nu \rho} D_{\rho} \bar{v}^{\mu}\right) \mathcal{T}_{\mu \nu}, \\
h^{\rho \mu} h^{\sigma \mu}\left(D_{\rho}-\mathcal{G}_{\rho}\right) \mathcal{T}_{\mu \nu} & =h^{\sigma \nu}\left[\bar{v}^{\mu} D_{\nu} \mathcal{P}_{\mu}-D_{\mu}\left(\bar{v}^{\mu} \mathcal{P}_{\nu}\right)+H_{\mu \nu} \mathcal{J}^{\mu}+K_{\mu \nu} \mathcal{E}^{\mu}\right]
\end{aligned}
$$

where

$$
\begin{aligned}
K_{\mu \nu} & =\partial_{\mu} \tau_{\nu}-\partial_{\nu} \tau_{\mu}, \\
\mathcal{G}_{\mu} & =T_{\mu \nu}^{\nu}=-K_{\mu \nu} \bar{v}^{\nu} .
\end{aligned}
$$

Invariance under the $\mathrm{U}(1)$ gauge symmetry of the gauge field $B_{\mu}$ gives the conservation equation of the mass current;

$$
\left(D_{\mu}-\mathcal{G}_{\mu}\right) \mathcal{J}^{\mu}=0
$$

Invariance under the Milne boost gives the following Ward identity;

$$
\mathcal{P}_{\mu}=\bar{h}_{\mu \nu} \mathcal{J}^{\nu} .
$$

The energy flow, momentum density and stress tensor are not invariant under the Milne boost. The variations are transforms under the Milne boost as

$$
\begin{aligned}
& \delta \bar{v}^{\prime \mu}=\delta \bar{v}^{\mu}+V_{\nu} \delta h^{\mu \nu}, \\
& \delta B_{\mu}^{\prime}=\delta B_{\mu}-\frac{1}{2} h^{\rho \nu} V_{\rho} V_{\nu} \delta \tau_{\mu}-\tau_{\mu}\left(V_{\nu} \delta \bar{v}^{\nu}+\frac{1}{2} V_{\nu} V_{\rho} \delta h^{\nu \rho}\right),
\end{aligned}
$$

where we imposed $V_{\mu} \bar{v}^{\mu}=0$ since $V_{\mu}$ always comes with $h^{\mu \nu}$ or $\bar{P}_{\nu}^{\mu}$. It should also be noted that the variation in (B.16) are constrained by the orthogonality (B.1) and normalization (B.3) conditions and hence have additional contribution to the variation of $\tau_{\mu}$;

$$
\begin{aligned}
\delta \bar{v}^{\mu} & =-\bar{v}^{\mu} \bar{v}^{\nu} \delta \tau_{\nu}+\bar{P}_{\nu}^{\mu} \delta \bar{v}^{\nu}, \\
\delta h^{\mu \nu} & =-\left(\bar{v}^{\mu} h^{\nu \rho}+\bar{v}^{\nu} h^{\mu \rho}\right) \delta \tau_{\rho}+\bar{P}_{\rho}^{\mu} \bar{P}_{\sigma}^{\nu} \delta h^{\rho \sigma},
\end{aligned}
$$

where the first terms in these equations are already included in (B.16) but their transformation gives additional terms in the transformation of the energy flow. Then, the transformation of the energy flow, momentum density and stress tensor are given by

$$
\begin{aligned}
\mathcal{E}^{\mu} & =\mathcal{E}^{\mu}-h^{\mu \rho} h^{\nu \sigma} \mathcal{T}_{\rho \nu} V_{\sigma}-\bar{v}^{\mu} h^{\nu \rho} \mathcal{P}_{\nu} V_{\rho}+\frac{1}{2} \mathcal{J}^{\mu} h^{\rho \sigma} V_{\rho} V_{\sigma}, \\
\mathcal{P}_{\mu}^{\prime} & =\mathcal{P}_{\mu}-\tau_{\mu} h^{\nu \rho} \mathcal{P}_{\nu} V_{\rho}-\tau_{\rho} \mathcal{J}^{\rho} \bar{P}_{\mu}^{\nu} V_{\nu}+\tau_{\mu} \tau_{\nu} \mathcal{J}^{\nu} h^{\rho \sigma} V_{\rho} V_{\sigma}, \\
\mathcal{T}^{\prime \mu \nu} & =\mathcal{T}^{\mu \nu}-\left(\mathcal{P}^{\mu} h^{\nu \rho}+\mathcal{P}^{\nu} h^{\mu \rho}\right) V_{\rho}+h^{\mu \rho} h^{\nu \sigma} V_{\rho} V_{\sigma} \tau_{\lambda} \mathcal{J}^{\lambda},
\end{aligned}
$$

where the indices are raised or lowered by the spatial metric $h^{\mu \nu}$ or $\bar{h}_{\mu \nu}$, respectively, and the transformation of the stress tensor is given by (B.29) with (B.15). The mass current is invariant under the Milne boost;

$$
\mathcal{J}^{\prime \mu}=\mathcal{J}^{\mu},
$$

and in fact, (B.28) is consistent with the Ward identity (B.22) with (B.15) and (B.30). 
We introduce Milne boost invariant combinations. It is straightforward to see that the following combination is invariant under the Milne boost;

$$
\widetilde{\mathcal{T}}^{\mu}{ }_{\nu}=\mathcal{J}^{\mu} \bar{v}^{\nu}+\bar{v}^{\mu} \mathcal{P}^{\nu}+\mathcal{T}^{\mu \nu}
$$

which is an analogue of the stress-energy tensor, but constructed from the mass current, momentum density and stress tensor. We can also define the stress-energy tensor from the energy flow, momentum density and stress tensor;

$$
\bar{T}^{\mu}{ }_{\nu}=-\mathcal{E}^{\mu} \tau_{\nu}+\bar{v}^{\mu} \mathcal{P}_{\nu}+h^{\mu \rho} \mathcal{T}_{\rho \nu}
$$

but this stress-energy tensor is not invariant under the Milne boost and transforms as

$$
\bar{T}^{\prime \mu}{ }_{\nu}=\bar{T}^{\mu}{ }_{\nu}-\mathcal{J}^{\mu} V_{\nu}+\frac{1}{2} \mathcal{J}^{\mu} \tau_{\nu} h^{\rho \sigma} V_{\rho} V_{\sigma} .
$$

With this stress-energy tensor, a Milne boost invariant combination is given by

$$
\widehat{T}^{\mu}{ }_{\nu}=\bar{T}_{\nu}^{\mu}+\mathcal{J}^{\mu} B_{\nu} .
$$

It should be noted that the Milne boost combination $\widehat{T}^{\mu}{ }_{\nu}$ is not invariant (or covariant) under the $\mathrm{U}(1)$ gauge transformation associated to $B_{\mu}$.

Now, we consider fluids in the Newton-Cartan theory. We introduce the fluid velocity field $u^{\mu}$, which is invariant under the Milne boost. For the first order fluid in the Eckart frame, the energy flow, momentum density, stress tensor and mass current are given by

$$
\begin{aligned}
\mathcal{E}^{\mu} & =\mathcal{E} u^{\mu}+\frac{1}{2} \rho u^{2} u^{\mu}-\kappa h^{\mu \nu} \partial_{\nu} T+h^{\mu \rho} u^{\sigma} \mathcal{T}_{\rho \sigma}, \\
\mathcal{P}_{\mu} & =\rho u_{\mu} \\
\mathcal{T}_{\mu \nu} & =P \bar{h}_{\mu \nu}+\rho u_{\mu} u_{\nu}-\eta \sigma_{\rho \sigma} \bar{P}_{\mu}^{\rho} \bar{P}_{\nu}^{\sigma}-\zeta \theta \bar{h}_{\mu \nu}, \\
\mathcal{J}^{\mu} & =\rho u^{\mu}
\end{aligned}
$$

where the index of the velocity field is lowered by using the spatial metric $\bar{h}_{\mu \nu}$ and $u^{2}$ is defined as

$$
\begin{aligned}
u_{\mu} & =\bar{h}_{\mu \nu} u^{\nu}, \\
u^{2} & =\bar{h}_{\mu \nu} u^{\mu} u^{\nu} .
\end{aligned}
$$

The energy density $\mathcal{E}$, pressure $P$ and mass density $\rho$ are invariant under the Milne boost. We construct a stress-energy tensor from the above quantities, and then $\bar{T}^{\mu}{ }_{\nu}$ is obtained as

$$
\begin{aligned}
\bar{T}_{\nu}^{\mu}= & -\mathcal{E} u^{\mu} \tau_{\nu}-\frac{1}{2} \rho u^{2} u^{\mu}+P \widehat{P}_{\nu}^{\mu}+\rho u^{\mu} u_{\nu} \\
& -\eta \sigma_{\rho \sigma} h^{\mu \rho} \widehat{P}_{\nu}^{\sigma}-\zeta \theta \widehat{P}_{\nu}^{\mu}+\kappa h^{\mu \rho}\left(\partial_{\rho} T\right) \tau_{\nu}
\end{aligned}
$$

where

$$
\widehat{P}_{\nu}^{\mu} \equiv \delta^{\mu}{ }_{\nu}-u^{\mu} \tau_{\nu}=\bar{P}_{\nu}^{\mu}-h^{\mu \rho} u_{\rho} \tau_{\nu}
$$


We consider the Milne boost invariants in fluid mechanics. We introduce a Milne boost invariant combination for the gauge field by using $u^{\mu}$;

$$
\widehat{B}=B+u_{\mu} d x^{\mu}-\frac{1}{2} u^{2} \tau_{\rho} d x^{\rho} .
$$

Then, the Milne boost invariant combination for the stress-energy tensor $\widehat{T}^{\mu}{ }_{\nu}$ is expressed in terms of the Milne boost invariant gauge field $\widehat{B}_{\mu}$ as

$$
\begin{aligned}
\widehat{T}^{\mu}{ }_{\nu} & =\bar{T}^{\mu}{ }_{\nu}+\mathcal{J}^{\mu} B_{\nu} \\
& =-\mathcal{E} u^{\mu} \tau_{\nu}+P \widehat{P}_{\nu}^{\mu}+\mathcal{J}^{\mu} \widehat{B}_{\nu}-\eta \sigma_{\rho \sigma} h^{\mu \rho} \widehat{P}_{\nu}^{\sigma}-\zeta \theta \widehat{P}_{\nu}^{\mu}+\kappa h^{\mu \rho}\left(\partial_{\rho} T\right) \tau_{\nu} .
\end{aligned}
$$

This stress-energy tensor is invariant under the Milne boost, but is not invariant under the $\mathrm{U}(1)$ gauge symmetry associated to $B_{\mu}$. We define the Milne boost invariant and U(1) gauge invariant stress-energy tensor, $T^{\mu}{ }_{\nu}$ from $\widehat{T}^{\mu}{ }_{\nu}$ and $\widehat{B}_{\mu}$ such that

$$
T_{\nu}^{\mu} \equiv \widehat{T}_{\nu}^{\mu}-\mathcal{J}^{\mu} \widehat{B}_{\nu}
$$

Then, it is expressed as

$$
T_{\nu}^{\mu}=-\mathcal{E} u^{\mu} \tau_{\nu}+P \widehat{P}_{\nu}^{\mu}-\eta \sigma_{\rho \sigma} h^{\mu \rho} \widehat{P}_{\nu}^{\sigma}-\zeta \theta \widehat{P}_{\nu}^{\mu}+\kappa h^{\mu \rho}\left(\partial_{\rho} T\right) \tau_{\nu}
$$

The Milne boost invariant stress-energy tensor (B.46) can be decomposed into the Milne boost invariant energy density $\widehat{\mathcal{E}}^{\mu}$, momentum density $\widehat{\mathcal{P}}_{\mu}$ and stress tensor $\widehat{\mathcal{T}}^{\mu}{ }_{\nu}$ as

$$
T^{\mu}{ }_{\nu}=-\widehat{\mathcal{E}}^{\mu} \tau_{\nu}+u^{\mu} \widehat{\mathcal{P}}_{\nu}+\widehat{\mathcal{T}}^{\mu}{ }_{\nu}
$$

where

$$
\begin{aligned}
\widehat{\mathcal{E}}^{\mu} & \equiv-T^{\mu}{ }_{\nu} u^{\nu}=\mathcal{E} u^{\mu}-\kappa h^{\mu \nu} \partial_{\nu} T \\
\widehat{\mathcal{P}}_{\mu} & \equiv T^{\rho}{ }_{\nu} \tau_{\rho} \widehat{P}_{\mu}^{\nu}=0 \\
\widehat{\mathcal{T}}^{\mu}{ }_{\nu} & \equiv T^{\rho}{ }_{\sigma} \widehat{P}_{\rho}^{\mu} \widehat{P}_{\nu}^{\sigma}=P \widehat{P}_{\nu}^{\mu}-\eta \sigma_{\rho \sigma} h^{\mu \rho} \widehat{P}_{\nu}^{\sigma}-\zeta \theta \widehat{P}_{\nu}^{\mu} .
\end{aligned}
$$

By using the relation between $T^{\mu}{ }_{\nu}$ and $\bar{T}^{\mu}{ }_{\nu}$;

$$
T^{\mu}{ }_{\nu}=\bar{T}^{\mu}{ }_{\nu}+\mathcal{J}^{\mu} B_{\nu}-\mathcal{J}^{\mu} \widehat{B}_{\nu}=\bar{T}^{\mu}{ }_{\nu}-\mathcal{J}^{\mu}\left(u_{\nu}-\frac{1}{2} \tau_{\nu} u^{2}\right),
$$

the Milne boost invariant energy flow, momentum density and stress tensor are expressed as

$$
\begin{aligned}
\widehat{\mathcal{E}}^{\mu} & =\mathcal{E}^{\mu}-\mathcal{T}^{\mu}{ }_{\nu} u^{\nu}-\bar{v}^{\mu} \mathcal{P}_{\nu} u^{\nu}+\frac{1}{2} u^{2} \mathcal{J}^{\mu}, \\
\widehat{\mathcal{P}}_{\mu} & =\left(\mathcal{P}_{\nu}-\tau_{\rho} \mathcal{J}^{\rho} u_{\nu}\right) \widehat{P}_{\mu}^{\nu}=0 \\
\widehat{\mathcal{T}}^{\mu}{ }_{\nu} & =h^{\mu \rho}\left(\mathcal{T}_{\rho \sigma}-\rho u_{\rho} u_{\sigma}\right) \widehat{P}_{\nu}^{\sigma}
\end{aligned}
$$

where we have used the condition for the Eckart frame (B.38) in the last equality of (B.53) and in (B.54). The Milne boost invariant energy flow (B.52) is equivalent to that introduced in $[70]$ 
In general, the Milne boost invariant quantities $\widehat{\mathcal{E}}^{\mu}, \widehat{\mathcal{P}}_{\mu}, \widehat{\mathcal{T}}^{\mu}{ }_{\nu}$ and $\widehat{T}^{\mu}{ }_{\nu}$ are different from the physical quantities $\mathcal{E}^{\mu}, \mathcal{P}_{\mu}, \mathcal{T}^{\mu}{ }_{\nu}$ and $T^{\mu}{ }_{\nu}$, but they agree if we take a special frame of the Milne boost transformation by imposing the condition

$$
\bar{v}^{\mu}=u^{\mu}
$$

and we can take this frame by using the Milne boost with

$$
V_{\mu}=\bar{h}_{\mu \nu}\left(u^{\nu}-\bar{v}^{\nu}\right)=u_{\mu} .
$$

This also implies that the conservation law for the Milne boost invariant quantities $\widehat{\mathcal{E}}^{\mu}, \widehat{\mathcal{P}}_{\mu}$ and $\widehat{\mathcal{T}}^{\mu}{ }_{\nu}$ is given by

$$
\begin{aligned}
\left(\widehat{D}_{\mu}-\widehat{\mathcal{G}}_{\mu}\right) \widehat{\mathcal{E}}^{\mu} & =u^{\mu}\left(\widehat{H}_{\mu \nu} \mathcal{J}^{\nu}+K_{\mu \nu} \widehat{\mathcal{E}}^{\nu}\right)-\frac{1}{2}\left(h^{\mu \rho} \widehat{D}_{\rho} u^{\nu}+h^{\nu \rho} \widehat{D}_{\rho} u^{\mu}\right) \widehat{\mathcal{T}}_{\mu \nu}, \\
h^{\rho \mu} h^{\sigma \nu}\left(\widehat{D}_{\rho}-\widehat{\mathcal{G}}_{\rho}\right) \widehat{\mathcal{T}}_{\mu \nu} & =h^{\sigma \nu}\left[u^{\mu} \widehat{D}_{\nu} \widehat{\mathcal{P}}_{\mu}-\widehat{D}_{\mu}\left(u^{\mu} \widehat{\mathcal{P}}_{\nu}\right)+\widehat{H}_{\mu \nu} \mathcal{J}^{\mu}+K_{\mu \nu} \widehat{\mathcal{E}}^{\mu}\right]
\end{aligned}
$$

where $\widehat{D}_{\mu}$ is the covariant derivative in this frame and

$$
\widehat{\mathcal{G}}_{\mu}=-K_{\mu \nu} u^{\mu} .
$$

We call this frame as "holographic frame," since the result is obtained in this frame if we consider the holographic duality for the Lifshitz spacetimes.

\section{Lifshitz fluid in scalar background}

In section 5, we have seen that the Lifshitz black hole geometry corresponds to fluids with a non-zero chemical potential but without non-trivial scalar background. On the other hand, the dimensional reduction from the higher dimensional model in section 6 gives fluids with zero chemical potential but with non-trivial scalar background. Here, we show that the results in section 5 can also be interpreted as fluids with zero chemical potential but with non-trivial scalar background, even though the pressure is different from that in first law of thermodynamics.

The stress-energy tensor $\widetilde{T}^{\mu}{ }_{\nu}$ can be expressed in the following form;

$$
\widetilde{T}^{\mu}{ }_{\nu}=-\mathcal{E} \hat{v}^{\mu} \tau_{\nu}+P \hat{h}^{\mu}{ }_{\nu}-\kappa \tau_{\nu} h^{\mu \rho} \partial_{\rho} T-\eta \sigma_{a b} e_{a}^{\mu} \hat{e}_{\nu}^{b}+n \hat{v}^{\mu} \mathcal{A}_{\nu}-n \hat{v}^{\rho} \mathcal{A}_{\rho} \delta^{\mu}{ }_{\nu},
$$

where the energy density $\mathcal{E}$ and particle number density $n$ have the same form (5.8), but the pressure is now given by

$$
P=\frac{z}{16 \pi G} a^{\frac{\theta}{3(3-\theta)}} r_{0}^{z+3-\theta} .
$$

The stress-energy tensor $\widetilde{T}^{\mu}{ }_{\nu}$ is related to that in the Newton-Cartan theory $T^{\mu}{ }_{\nu}$ by the same equation (5.46), but now the relation between $\mathcal{A}$ and $\widehat{B}$ is given by

$$
\mathcal{A}_{\mu}=m \widehat{B}_{\mu}
$$

which implies that $\mu=0$. 
The fluid equations are expressed as

$$
\begin{aligned}
& 0=\partial_{t} \mathcal{E}+v^{i} \partial_{i} \mathcal{E}+(\mathcal{E}+P) \partial_{i} v^{i} \\
& -\frac{1}{2} \eta \sigma_{i j} \sigma_{i j}-\partial_{i}\left(\kappa \partial_{i} T\right)+c_{\phi}\left\langle\widetilde{\mathcal{O}}_{\phi}\right\rangle \frac{1}{n}\left(\partial_{t} n+v^{i} \partial_{i} n\right), \\
& 0=\partial_{i} P+n \mathcal{F}_{t i}+n v^{j} \mathcal{F}_{j i}-\partial_{j}\left(\eta \sigma_{i j}\right)-c_{\phi}\left\langle\widetilde{\mathcal{O}}_{\phi}\right\rangle \frac{1}{n} \partial_{i} n, \\
& 0=\partial_{t} n+\partial_{j}\left(n v^{j}\right) \text {, }
\end{aligned}
$$

where $c_{\phi}$ is the coupling constant between $\widetilde{\mathcal{O}}_{\phi}$ and $\log a$, which is given by

$$
c_{\phi}=-\frac{\theta}{3}\left\{\frac{9(z-1)+(3-\theta) \theta}{\sqrt{6(3-\theta)[3(z-1)-\theta]}}\right\}^{-1},
$$

and the operator $\widetilde{\mathcal{O}}_{\phi}$ is the dual of the dilaton $\phi$ but we have removed the contribution form the external gauge field as for the stress-energy tensor;

$$
\left\langle\widetilde{\mathcal{O}}_{\phi}\right\rangle=\left\langle\mathcal{O}_{\phi}\right\rangle-\left(\lambda-\frac{1}{2} \nu\right) J^{\mu} \mathcal{A}_{\mu} .
$$

where $\lambda$ and $\nu$ are defined in (2.11) and (2.12) respectively.

The additional term associated to $\widetilde{\mathcal{O}}_{\phi}$ can be understood as follows. In terms of the boundary stress-energy tensor, (C.4) and (C.5) are expressed as

$$
D_{\mu} \mathcal{T}^{\mu}{ }_{\nu}=\mathcal{F}_{\mu \nu} J^{\mu}+\bar{c}_{\phi}\left\langle\widetilde{\mathcal{O}}_{\phi}\right\rangle \partial_{\nu} \phi_{0},
$$

where $\phi_{0}$ is the constant mode of the dilaton $\phi$, which is defined in (2.13) and is interpreted as the source for $\widetilde{O}_{\phi}$. The coupling constant $\bar{c}_{\phi}$ is now redefined as

$$
\bar{c}_{\phi}=-\frac{(3-\theta) \theta}{9(z-1)+(3-\theta) \theta} .
$$

This additional term implies the presence of an external force whose potential is proportional to the particle number density. Although, $\phi_{0}$ is interpreted as the external field, it is related to the particle number density $n$, or equivalently $a$, as seen from (2.13).

For the fluid dual to the Lifshitz geometry, the energy density is constrained first by the Lifshitz Ward identity with hyperscaling violation. It is given by

$$
-\left(z-\frac{\theta}{3}\right) \mathcal{E}+(3-\theta) P-(3-\theta) c_{\phi}\left\langle\widetilde{\mathcal{O}}_{\phi}\right\rangle=0,
$$

where the operator $\widetilde{\mathcal{O}}_{\phi}$ and coupling constant $c_{\phi}$ are defined by (C.8) and (C.7), respectively. The coefficients of $\mathcal{E}, P$ and $c_{\phi}\left\langle\widetilde{\mathcal{O}}_{\phi}\right\rangle$ are the scaling dimensions of $t, x^{i}$ and $a$ with appropriate signs, respectively. (See appendix D for more details on the scaling dimensions for the Lifshitz symmetry with hyperscaling-violation.)

The thermodynamics relation given by

$$
T J_{S}^{\mu}=\widehat{\mathcal{E}}^{\mu}+P \hat{v}^{\mu}=-T^{\mu}{ }_{\nu} \hat{v}^{\nu}+P \hat{v}^{\mu} .
$$

This is consistent with $\mu=0$. 


\section{Scaling dimensions in fluids}

If there are no hyperscaling-violation, the Lifshitz spacetime is invariant under the following scaling transformation;

$$
t \rightarrow c^{z} t, \quad \quad x^{i} \rightarrow c x^{i}, \quad r \rightarrow c^{-1} r .
$$

However, in the presence of the hyperscaling-violation, the following metric and gauge field are not invariant under the above transformation;

$$
\begin{aligned}
d s^{2} & =b^{-\frac{2 \theta}{(d-1)(d-1-\theta)}} r^{-2 \theta /(d-1)}\left(-r^{2 z} d t^{2}+\frac{d r^{2}}{r^{2}}+\sum_{i} r^{2}\left(d x^{i}\right)^{2}\right) \\
A & =a r^{z+d-1-\theta} d t
\end{aligned}
$$

but transform as

$$
d s^{2} \rightarrow c^{2 \theta /(d-1)} d s^{2}, \quad A \rightarrow c^{-(d-1)+\theta} A .
$$

where $a, b$ are constant parameters above. However, if we impose the following scaling behavior to the constant parameters

$$
a \rightarrow c^{d-1-\theta} a, \quad b \rightarrow c^{d-1-\theta} b,
$$

then the metric and gauge field are invariant under the scaling transformation. For the black hole geometry, the horizon radius transforms as

$$
r_{0} \rightarrow c^{-1} r_{0}
$$

Then, the coordinates and the constants have the following scaling dimensions:

$$
\begin{aligned}
& {[t]=-z, \quad\left[x^{i}\right]=-1,} \\
& {[a]=-(d-1)+\theta, \quad[b]=-(d-1)+\theta, \quad\left[r_{0}\right]=1 .}
\end{aligned}
$$

After the redefinition of the coordinate (3.10), the scaling dimension of the coordinates becomes

$$
[t]=-z+\frac{\theta}{d-1}, \quad\left[x^{i}\right]=-1+\frac{\theta}{d-1} .
$$

Then, the scaling dimension of the velocity field should be

$$
\left[v^{i}\right]=z-1 .
$$

The constant modes of the gauge field have the following dimensions

$$
\left[\mathcal{A}_{t}\right]=z-\frac{\theta}{d-1}, \quad\left[\mathcal{A}_{i}\right]=1-\frac{\theta}{d-1} .
$$


¿From (5.8) and (3.13), or (6.101) and (6.24), the scaling dimensions of the energy density, pressure, the particle number density and temperature are given by

$$
\begin{array}{ll}
{[\mathcal{E}]=z+(d-1)-\frac{d}{d-1} \theta,} & {[P]=z+(d-1)-\frac{d}{d-1} \theta,} \\
{[n]=d-1-\theta,} & {[T]=z-\frac{\theta}{d-1} .}
\end{array}
$$

They satisfies the appropriate relation to the dimension of the coordinates

$$
[\mathcal{E}]=-[t]-(d-1)\left[x^{i}\right], \quad[n]=-(d-1)\left[x^{i}\right], \quad[T]=-[t],
$$

namely, dimensions of the density is minus of dimension of volume, and dimensions of the energy and temperature are minus of the dimension of time. From the relation to fluid variables, the scaling dimensions of the transport coefficients are calculated as

$$
[\eta]=d-1-\theta, \quad[\zeta]=d-1-\theta, \quad[\kappa]=z-1-\frac{d-2}{d-1} \theta,
$$

which are consistent with (5.13). Since the gauge field in the Newton-Cartan theory

$$
\left[B_{t}\right]=2 z-2, \quad\left[B_{i}\right]=z-1,
$$

the scaling dimension of mass $m$ is given by

$$
[m]=-z+2-\frac{\theta}{d-1} .
$$

Open Access. This article is distributed under the terms of the Creative Commons Attribution License (CC-BY 4.0), which permits any use, distribution and reproduction in any medium, provided the original author(s) and source are credited.

\section{References}

[1] E. Kiritsis and Y. Matsuo, Charge-hyperscaling violating Lifshitz hydrodynamics from black-holes, JHEP 12 (2015) 076 [arXiv: 1508.02494] [INSPIRE].

[2] J.M. Maldacena, The large-N limit of superconformal field theories and supergravity, Int. J. Theor. Phys. 38 (1999) 1113 [Int. J. Theor. Phys. 38 (1999) 1113] [hep-th/9711200] [INSPIRE].

[3] S.S. Gubser, I.R. Klebanov and A.M. Polyakov, Gauge theory correlators from noncritical string theory, Phys. Lett. B 428 (1998) 105 [hep-th/9802109] [INSPIRE].

[4] E. Witten, Anti-de Sitter space and holography, Adv. Theor. Math. Phys. 2 (1998) 253 [hep-th/9802150] [INSPIRE].

[5] E. Witten, Anti-de Sitter space, thermal phase transition and confinement in gauge theories, Adv. Theor. Math. Phys. 2 (1998) 505 [hep-th/9803131] [InSPIRE].

[6] D.T. Son and A.O. Starinets, Minkowski space correlators in AdS/CFT correspondence: Recipe and applications, JHEP 09 (2002) 042 [hep-th/0205051] [INSPIRE].

[7] G. Policastro, D.T. Son and A.O. Starinets, From AdS/CFT correspondence to hydrodynamics, JHEP 09 (2002) 043 [hep-th/0205052] [INSPIRE]. 
[8] G. Policastro, D.T. Son and A.O. Starinets, From AdS/CFT correspondence to hydrodynamics. 2. Sound waves, JHEP 12 (2002) 054 [hep-th/0210220] [INSPIRE].

[9] R. Baier, P. Romatschke, D.T. Son, A.O. Starinets and M.A. Stephanov, Relativistic viscous hydrodynamics, conformal invariance and holography, JHEP 04 (2008) 100 [arXiv: 0712.2451] [INSPIRE].

[10] S. Bhattacharyya, V.E. Hubeny, S. Minwalla and M. Rangamani, Nonlinear fluid dynamics from gravity, JHEP 02 (2008) 045 [arXiv:0712.2456] [INSPIRE].

[11] S. Bhattacharyya et al., Local fluid dynamical entropy from gravity, JHEP 06 (2008) 055 [arXiv:0803.2526] [INSPIRE].

[12] D.T. Son, Toward an AdS/cold atoms correspondence: a geometric realization of the Schrödinger symmetry, Phys. Rev. D 78 (2008) 046003 [arXiv: 0804.3972] [InSPIRE].

[13] K. Balasubramanian and J. McGreevy, Gravity duals for non-relativistic CFTs, Phys. Rev. Lett. 101 (2008) 061601 [arXiv:0804.4053] [INSPIRE].

[14] S. Kachru, X. Liu and M. Mulligan, Gravity duals of Lifshitz-like fixed points, Phys. Rev. D 78 (2008) 106005 [arXiv: 0808.1725] [INSPIRE].

[15] M. Taylor, Non-relativistic holography, arXiv:0812.0530 [INSPIRE].

[16] M. Guica, K. Skenderis, M. Taylor and B.C. van Rees, Holography for Schrödinger backgrounds, JHEP 02 (2011) 056 [arXiv: 1008.1991] [INSPIRE].

[17] C. Charmousis, B. Gouteraux, B.S. Kim, E. Kiritsis and R. Meyer, Effective holographic theories for low-temperature condensed matter systems, JHEP 11 (2010) 151 [arXiv: 1005.4690] [INSPIRE].

[18] B. Gouteraux and E. Kiritsis, Generalized holographic quantum criticality at finite density, JHEP 12 (2011) 036 [arXiv:1107.2116] [INSPIRE].

[19] B. Gouteraux and E. Kiritsis, Quantum critical lines in holographic phases with (un)broken symmetry, JHEP 04 (2013) 053 [arXiv:1212.2625] [INSPIRE].

[20] M. Taylor, Lifshitz holography, Class. Quant. Grav. 33 (2016) 033001 [arXiv:1512.03554] [INSPIRE].

[21] D.M. Eagles, Possible pairing without superconductivity at low carrier concentrations in bulk and thin-film superconducting semiconductors, Phys. Rev. 186 (1969) 456 [INSPIRE].

[22] P. Nozieres and S. Schmitt-Rink, Bose condensation in an attractive fermion gas: From weak to strong coupling superconduct ivity, J. Low. Temp. Phys. 59 (1985) 195 [InSPIRE].

[23] K.M. O'Hara, S.L. Hemmer, M.E. Gehm, S.R. Granade and J.E. Thomas, Observation of a strongly interacting degenerate Fermi gas of atoms, Science 298 (2002) 2179 [cond-mat/0212463] [INSPIRE].

[24] C.A. Regal, M. Greiner and D.S. Jin, Observation of resonance condensation of fermionic atom pairs, Phys. Rev. Lett. 92 (2004) 040403 [INSPIRE].

[25] M. Bartenstein et al., Crossover from a molecular Bose-Einstein condensate to a degenerate Fermi gas, Phys. Rev. Lett. 92 (2004) 120401 [inSPIRE].

[26] A.A. Patel, A. Eberlein and S. Sachdev, Shear viscosity at the Ising-nematic quantum critical point in two dimensional metals, Phys. Rev. B 95 (2017) 075127 [arXiv:1607.03894] [INSPIRE].

[27] R.M. Hornreich, M. Luban and S. Shtrikman, Critical behavior at the onset of $k$-space instability on the $\lambda$ line, Phys. Rev. Lett. 35 (1975) 1678 [INSPIRE]. 
[28] T. Mehen, I.W. Stewart and M.B. Wise, Conformal invariance for nonrelativistic field theory, Phys. Lett. B 474 (2000) 145 [hep-th/9910025] [InSPIRE].

[29] E. Ardonne, P. Fendley and E. Fradkin, Topological order and conformal quantum critical points, Annals Phys. 310 (2004) 493 [cond-mat/0311466] [INSPIRE].

[30] C.P. Herzog, M. Rangamani and S.F. Ross, Heating up Galilean holography, JHEP 11 (2008) 080 [arXiv: 0807.1099] [INSPIRE].

[31] M. Rangamani, S.F. Ross, D.T. Son and E.G. Thompson, Conformal non-relativistic hydrodynamics from gravity, JHEP 01 (2009) 075 [arXiv:0811.2049] [INSPIRE].

[32] D.K. Brattan, Charged, conformal non-relativistic hydrodynamics, JHEP 10 (2010) 015 [arXiv: 1003.0797] [INSPIRE].

[33] C. Hoyos, B.S. Kim and Y. Oz, Lifshitz field theories at non-zero temperature, hydrodynamics and gravity, JHEP 03 (2014) 029 [arXiv:1309.6794] [INSPIRE].

[34] C. Hoyos, B.S. Kim and Y. Oz, Bulk viscosity in holographic Lifshitz hydrodynamics, JHEP 03 (2014) 050 [arXiv: 1312.6380] [InSPIRE].

[35] C. Cao et al., Universal quantum viscosity in a unitary Fermi gas, Science 331 (2011) 58 [arXiv: 1007.2625] [INSPIRE].

[36] D. Forcella, J. Zaanen, D. Valentinis and D. van der Marel, Electromagnetic properties of viscous charged fluids, Phys. Rev. B 90 (2014) 035143 [arXiv: 1406.1356] [INSPIRE].

[37] M. Muller and S. Sachdev, Collective cyclotron motion of the relativistic plasma in graphene, Phys. Rev. B 78 (2008) 115419 [arXiv:0801.2970] [InSPIRE].

[38] L. Fritz, J. Schmalian, M. Müller and S Sachdev, Quantum critical transport in clean graphene, Phys. Rev. B 78 (2008) 085416 [arXiv:0802.4289].

[39] M. Mueller, J. Schmalian and L. Fritz, Graphene - A nearly perfect fluid, Phys. Rev. Lett. 103 (2009) 025301 [arXiv:0903.4178].

[40] D.A. Bandurin et al., Negative local resistance caused by viscous electron backflow in graphene, Science 351 (2016) 1055 [arXiv: 1509.04165].

[41] J. Crossno et al., Observation of the Dirac fluid and the breakdown of the Wiedemann-Franz law in graphene, Science 351 (2016) 1058 [arXiv: 1509.04713].

[42] P.J.W. Moll et al., Evidence for hydrodynamic electron flow in $\mathrm{PdCoO}_{2}$, Science 351 (2016) 1061 [arXiv: 1509.05691].

[43] I. Torre, A. Tomadin, A.K. Geim and M. Polini, Nonlocal transport and the hydrodynamic shear viscosity in graphene, Phys. Rev. B 92 (2015) 165433 [arXiv:1508.00363] [INSPIRE].

[44] L. Levitov and G. Falkovich, Electron viscosity, current vortices and negative nonlocal resistance in graphene, Nature Phys. 12 (2016) 672 [arXiv:1508.00836].

[45] A. Lucas, J. Crossno, K.C. Fong, P. Kim and S. Sachdev, Transport in inhomogeneous quantum critical fluids and in the Dirac fluid in graphene, Phys. Rev. B 93 (2016) 075426 [arXiv: 1510.01738] [INSPIRE].

[46] J. Gath, J. Hartong, R. Monteiro and N.A. Obers, Holographic models for theories with hyperscaling violation, JHEP 04 (2013) 159 [arXiv:1212.3263] [INSPIRE].

[47] B. Goutéraux, Universal scaling properties of extremal cohesive holographic phases, JHEP 01 (2014) 080 [arXiv: 1308.2084] [INSPIRE].

[48] A. Karch, Conductivities for hyperscaling violating geometries, JHEP 06 (2014) 140 [arXiv: 1405.2926] [INSPIRE]. 
[49] L. Huijse, S. Sachdev and B. Swingle, Hidden Fermi surfaces in compressible states of gauge-gravity duality, Phys. Rev. B 85 (2012) 035121 [arXiv:1112.0573] [INSPIRE].

[50] D.T. Son, Newton-Cartan geometry and the quantum Hall effect, arXiv:1306.0638 [INSPIRE].

[51] M.H. Christensen, J. Hartong, N.A. Obers and B. Rollier, Torsional Newton-Cartan geometry and Lifshitz holography, Phys. Rev. D 89 (2014) 061901 [arXiv:1311.4794] [InSPIRE].

[52] M.H. Christensen, J. Hartong, N.A. Obers and B. Rollier, Boundary stress-energy tensor and Newton-Cartan geometry in Lifshitz holography, JHEP 01 (2014) 057 [arXiv:1311.6471] [INSPIRE].

[53] E.A. Bergshoeff, J. Hartong and J. Rosseel, Torsional Newton-Cartan geometry and the Schrödinger algebra, Class. Quant. Grav. 32 (2015) 135017 [arXiv: 1409.5555] [INSPIRE].

[54] J. Hartong, E. Kiritsis and N.A. Obers, Lifshitz space-times for Schrödinger holography, Phys. Lett. B 746 (2015) 318 [arXiv:1409.1519] [INSPIRE].

[55] J. Hartong, E. Kiritsis and N.A. Obers, Schrödinger invariance from Lifshitz isometries in holography and field theory, Phys. Rev. D 92 (2015) 066003 [arXiv:1409.1522] [InSPIRE].

[56] J. Hartong, E. Kiritsis and N.A. Obers, Field theory on Newton-Cartan backgrounds and symmetries of the Lifshitz vacuum, JHEP 08 (2015) 006 [arXiv: 1502.00228] [INSPIRE].

[57] S.F. Ross and O. Saremi, Holographic stress tensor for non-relativistic theories, JHEP 09 (2009) 009 [arXiv:0907.1846] [InSPIRE].

[58] W. Chemissany and I. Papadimitriou, Lifshitz holography: the whole shebang, JHEP 01 (2015) 052 [arXiv: 1408.0795] [INSPIRE].

[59] W. Chemissany and I. Papadimitriou, Generalized dilatation operator method for non-relativistic holography, Phys. Lett. B 737 (2014) 272 [arXiv:1405.3965] [INSPIRE].

[60] U. Gürsoy, A. Jansen, W. Sybesma and S. Vandoren, Holographic equilibration of nonrelativistic plasmas, Phys. Rev. Lett. 117 (2016) 051601 [arXiv:1602.01375] [INSPIRE].

[61] J. Hartong, N.A. Obers and M. Sanchioni, Lifshitz hydrodynamics from Lifshitz black branes with linear momentum, JHEP 10 (2016) 120 [arXiv:1606.09543] [INSPIRE].

[62] E. Kiritsis, Supergravity, D-brane probes and thermal super Yang-Mills: a comparison, JHEP 10 (1999) 010 [hep-th/9906206] [INSPIRE].

[63] S.S. Gubser, Time warps, JHEP 01 (2010) 020 [arXiv:0812.5107] [INSPIRE].

[64] S.S. Gubser and A. Nellore, Ground states of holographic superconductors, Phys. Rev. D 80 (2009) 105007 [arXiv:0908.1972] [INSPIRE].

[65] S. Kuperstein and A. Mukhopadhyay, The unconditional RG flow of the relativistic holographic fluid, JHEP 11 (2011) 130 [arXiv:1105.4530] [INSPIRE].

[66] S. Kuperstein and A. Mukhopadhyay, Spacetime emergence via holographic RG flow from incompressible Navier-Stokes at the horizon, JHEP 11 (2013) 086 [arXiv:1307.1367] [INSPIRE].

[67] N. Behr, S. Kuperstein and A. Mukhopadhyay, Holography as a highly efficient renormalization group flow. I. Rephrasing gravity, Phys. Rev. D 94 (2016) 026001 [arXiv: 1502.06619] [INSPIRE].

[68] S.F. Ross, Holography for asymptotically locally Lifshitz spacetimes, Class. Quant. Grav. 28 (2011) 215019 [arXiv:1107.4451] [INSPIRE]. 
[69] P. Kovtun, D.T. Son and A.O. Starinets, Viscosity in strongly interacting quantum field theories from black hole physics, Phys. Rev. Lett. 94 (2005) 111601 [hep-th/0405231] [INSPIRE].

[70] K. Jensen, Aspects of hot Galilean field theory, JHEP 04 (2015) 123 [arXiv:1411.7024] [INSPIRE].

[71] K. Jensen, On the coupling of Galilean-invariant field theories to curved spacetime, arXiv:1408.6855 [INSPIRE].

[72] I. Kanitscheider and K. Skenderis, Universal hydrodynamics of non-conformal branes, JHEP 04 (2009) 062 [arXiv: 0901.1487] [inSPIRE].

[73] B. Gouteraux, J. Smolic, M. Smolic, K. Skenderis and M. Taylor, Holography for Einstein-Maxwell-dilaton theories from generalized dimensional reduction, JHEP 01 (2012) 089 [arXiv: 1110.2320] [INSPIRE].

[74] J. Hartong and N.A. Obers, Hořava-Lifshitz gravity from dynamical Newton-Cartan geometry, JHEP 07 (2015) 155 [arXiv:1504.07461] [INSPIRE].

[75] Y. Nakayama, Intrinsic ambiguity in second order viscosity parameters in relativistic hydrodynamics, Int. J. Mod. Phys. A 27 (2012) 1250125 [arXiv:1206.2421] [INSPIRE]. 